\title{
TESTE DE CONDUTIVIDADE ELÉTRICA NA AVALIAÇÃO DO VIGOR DE SEMENTES DE RÚCULA ${ }^{1}$
}

\author{
CHARLINE ZARATIN ALVES², MARCO EUSTÁQUIO DE SÁ3
}

\begin{abstract}
RESUMO - O trabalho teve por objetivo estudar metodologias para a condução do teste de condutividade elétrica, verificando sua eficiência na identificação de diferentes níveis de vigor de lotes de sementes de rúcula. Para tanto, o estudo foi conduzido utilizando cinco lotes de sementes de Rúcula Cultivada e cinco lotes de sementes de Rúcula Gigante. Foram realizados os testes de germinação, primeira contagem de germinação, emergência de plântulas em casa de vegetação e variações no teste de condutividade elétrica (temperaturas de $20^{\circ} \mathrm{C}, 25^{\circ} \mathrm{C}$ e $30^{\circ} \mathrm{C}$; volumes de $25 \mathrm{~mL}$, $50 \mathrm{~mL}$ e $75 \mathrm{~mL}$ de água; 25 e 50 sementes; e períodos de 1, 2, 4, 6, 8, 12, 16 e 24 horas). Diante dos resultados obtidos, verificou-se que o tempo de embebição para o teste de condutividade elétrica pode ser reduzido para 4 horas e o volume de água utilizado pode ser reduzido para $25 \mathrm{ou} 50 \mathrm{~mL}$. As combinações de 50 sementes apresentaram menor coeficiente de variação e as temperaturas mais adequadas foram de $25^{\circ} \mathrm{C}$ e $30^{\circ} \mathrm{C}$. Assim, concluiu-se que a condição mais adequada para o teste de condutividade elétrica em sementes de rúcula é a utilização de 50 sementes em $50 \mathrm{~mL}$ de água por 4 horas, a $25^{\circ} \mathrm{C}$.
\end{abstract}

Termos para indexação: Eruca sativa, potencial fisiológico, condutividade elétrica, qualidade de sementes.

\section{ELECTRICAL CONDUCTIVITY IN ARUGULA SEEDS}

\begin{abstract}
The research studied methodologies for the electrical conductivity test, to verify its sensitivity for identifying different vigor levels in arugula seed lots. Five seed lots each from the Rucula Cultivada and Rucula Gigante were used. The following tests were employed: standard germination, first germination count, seedling emergence and variations in the electrical conductivity test ( 25 or 50 seeds imbibed in $25 \mathrm{~mL}, 50 \mathrm{~mL}$ or $75 \mathrm{~mL}$ in water; at $20 \mathrm{oC}, 25 \mathrm{oC}$ and $30 \mathrm{oC}$; for $1,2,4,6,8,12,16$ and 24 hours). It was observed that the seed soaking period can be reduced to 4 hours and the water volume can be reduced to 25 or $50 \mathrm{~mL}$. The use of 50 seeds presented a lower variation coefficient and the best temperatures were $25 \mathrm{oC}$ and $30 \mathrm{oC}$. It was concluded that the electrical conductivity test for arugula seeds can be run using 50 seeds in $50 \mathrm{~mL}$ of water for 4 hours, at $25 \mathrm{oC}$.
\end{abstract}

Index terms: Eruca sativa, physiological potential, electrical conductivity, seed quality.

${ }^{1}$ Submetido em 27/08/2007. Aceito para publicação em 19/03/2008.

${ }^{2}$ Eng. Agr., Pós graduanda em Sistemas de Produção, UNESP, CEP 15385000. Ilha Solteira, SP. czaratin@bol.com.br
${ }^{3}$ Eng. Agr., Dr., Professor Titular, Departamento de Fitotecnia, Tecnologia de Alimentos e Sócio-Economia, UNESP, CEP 15385-000. Ilha Solteira, SP. mesa@agr.feis.unesp.br 


\section{INTRODUÇÃO}

Nos últimos anos, a produção de sementes de hortaliças no Brasil, teve uma demanda crescente por produtos de melhor qualidade e, em conseqüência, a forma de produção foi significativamente afetada. Apesar dos avanços, muito ainda há por fazer, não só para alcançar a auto suficiência em relação à produção, mas também em relação à obtenção de sementes de qualidade superior. A alta qualidade de sementes é de grande importância, principalmente quanto à germinação uniforme, necessária para garantir um estande ideal de plantas. Neste contexto, sementes de alto vigor se constituem em elemento básico e fundamental. Diante da crescente evolução, o aprimoramento dos testes de vigor empregados para diferenciar variações sutis na qualidade de sementes de espécies olerícolas, se faz necessário (Mendonça et al., 2003).

Assim sendo, além da necessidade de padronização de metodologias e interpretação dos resultados, os testes de vigor devem apresentar relação com a emergência de plântulas em campo, rapidez, objetividade, simplicidade, baixo custo e reprodutibilidade (AOSA, 1983).

Dentre os testes rápidos, o de condutividade elétrica tem sido bastante utilizado para a avaliação do vigor das sementes (Hampton, 1992) com resultados consistentes, principalmente para sementes de ervilha (Hampton e Tekrony, 1995), milho (Fagioli, 1997) e soja (Vieira et al., 1999).

A maioria dos estudos sobre o teste de condutividade elétrica tem sido realizada com espécies de sementes relativamente grandes (ISTA, 1995). Em sementes menores, como as de hortaliças, há necessidade de ajustar a metodologia para se obter informações confiáveis, já que diversos fatores podem interferir nos resultados. Dentre estes fatores destacam-se: características, tamanho das sementes e ocorrência de danificações (Tao, 1978), número de sementes avaliadas (Panobianco, 2000), genótipo (Rodo, 2002), tratamento químico da semente (Zhang e Hampton, 1999), além da temperatura durante a embebição e avaliação (Panobianco, 2000), quantidade de água (Rodo, 2002), período de embebição (Panobianco, 2000; Rodo, 2002) e teor de água das sementes (Vieira et al., 2002).

O teste de condutividade elétrica para avaliação do vigor de sementes de hortaliças ainda carece de uma definição de protocolo para cada espécie. Em pesquisa com sementes de cebola (Lima, 1993) não foi verificado sensibilidade do teste para separação de lotes de alto e baixo vigor. Para sementes de pimentão (Torres, 1996), dentre os testes de vigor avaliados, o de condutividade elétrica não se relacionou com a emergência das plântulas em campo. Em sementes de melão, Torres (2002) apontou que o teste de condutividade elétrica não se constituiu em opção eficiente para avaliação do vigor das sementes. Carpi (2005) verificou que esse teste pode se constituir em alternativa promissora para avaliação do vigor de sementes de rabanete, necessitando de estudos adicionais.

Por outro lado, Dias et al. (1996) em sementes de couveflor, cebola e cenoura, e Sá (1999) em sementes de tomate, constataram que o teste permitiu a separação dos lotes em diferentes níveis de vigor e demonstraram também, a possibilidade de redução do período de condicionamento das sementes. Andrade et al. (1995), em sementes de cenoura, também verificaram que o teste de condutividade elétrica foi o mais indicado para avaliar o vigor, em razão de sua facilidade de execução, objetividade e rapidez.

Para algumas espécies de hortaliças, nota-se que o teste de condutividade elétrica tem sido apontado como eficiente na avaliação do vigor, permitindo a identificação segura de diferenças no potencial fisiológico das sementes. No entanto, para sementes de rúcula, não há na literatura informações sobre metodologia específica para condução deste teste, justificando, portanto, a necessidade de intensificação da pesquisa para esta espécie.

\section{MATERIAL E MÉTODOS}

O trabalho foi conduzido no Laboratório de Produção e Tecnologia de Sementes da Universidade Estadual Paulista "Júlio de Mesquita Filho" - UNESP - do Campus de Ilha Solteira/SP, utilizando-se 5 lotes de sementes de duas cultivares de rúcula, no período de janeiro a agosto de 2007. Foram determinados o grau de umidade, porcentagem de germinação, emergência de plântulas e procedimentos específicos para a condução do teste de condutividade elétrica.

As sementes recebidas estavam embaladas em recipientes herméticos do tipo lata, e durante todo o período experimental, as mesmas permaneceram armazenadas em câmara seca a $20^{\circ} \mathrm{C}$ e $50 \%$ de umidade relativa do ar.

Determinação do grau de umidade: foi realizada pelo do método da estufa, durante 24 horas, a $105+/-3^{\circ} \mathrm{C}$, de acordo com as Regras para Análise de Sementes (Brasil, 1992). Foi utilizado duas amostras com aproximadamente, 2,0g de sementes para cada lote.

Teste de Germinação: foram utilizadas quatro repetições de 50 sementes por lote, distribuídas sobre uma folha de papel 
mata-borrão, previamente umedecidas com quantidade de água equivalente a 2,5 vezes o peso do substrato, colocadas no interior de caixas plásticas transparentes $(11,5 \times 11,5 \times$ $3,5 \mathrm{~cm}$ ), sendo mantidas em germinador a $25^{\circ} \mathrm{C}$. As avaliações foram realizadas aos quatro e sete dias após a semeadura, segundo os critérios estabelecidos pelas Regras para Análise de Sementes (Brasil, 1992).

Primeira Contagem de Germinação: realizada juntamente com o teste de germinação, computando-se a porcentagem média de plântulas normais, obtidas aos quatro dias após a semeadura.

Emergência de plântulas: foram utilizadas bandejas de isopor com 128 células individuais, contendo substrato comercial Plantmax Hortaliças. As bandejas foram mantidas em casa de vegetação com controle de temperatura $\left(25^{\circ} \mathrm{C}\right)$, com irrigações diárias ( 3 vezes ao dia). Foram utilizadas quatro repetições de 50 sementes por lote, colocando-se uma semente por célula. A avaliação da emergência das plântulas foi efetuada aos 10 dias após a semeadura, mediante a contagem de plântulas normais emergidas, avaliadas de acordo com os critérios adotados para avaliação da parte aérea de plântulas normais em um teste de germinação.

Condutividade elétrica: foram estudados os efeitos do período de embebição $(1,2,4,6,8,12,16,20$ e 24 horas), do volume de água destilada $(25,50$ e $75 \mathrm{~mL})$, do número de sementes (25 e 50$)$ e da temperatura de embebição $\left(20^{\circ} \mathrm{C}\right.$, $25^{\circ} \mathrm{C}$ e $30^{\circ} \mathrm{C}$ ). Foram utilizadas quatro subamostras de sementes fisicamente puras para cada lote. As sementes foram pesadas em balança de precisão de $0,0001 \mathrm{~g}$ e colocadas para embeber em copos plásticos contendo água destilada, sendo os mesmos mantidos em germinador durante cada período de embebição, dentro de cada temperatura estudada. As leituras da condutividade elétrica foram realizadas em condutivímetro Digimed DM-31, e os valores médios obtidos para cada lote, expressos em $\mu \mathrm{S} . \mathrm{cm}^{-1} \cdot \mathrm{g}^{-1}$ de semente.

Utilizou-se o delineamento experimental inteiramente casualizado, com quatro repetições, realizando-se a comparação das médias pelo teste de Tukey, em 5\% de probabilidade.

\section{RESULTADOS E DISCUSSÃO}

A avaliação inicial da qualidade fisiológica dos lotes de sementes de rúcula foi efetuada através dos testes de germinação, primeira contagem de germinação e emergência de plântulas em casa de vegetação. Nessa etapa, também foi avaliado o grau de umidade das sementes. Os resultados dessas avaliações se encontram na Tabela 1.

TABELA 1. Qualidade inicial de cinco lotes das cultivares Rúcula Cultivada e Rúcula Gigante.

\begin{tabular}{|c|c|c|c|c|c|}
\hline Cultivares & Lotes & Umidade & Germinação & $\begin{array}{l}\text { Primeira } \\
\text { Contagem }\end{array}$ & $\begin{array}{l}\text { Emergência de } \\
\text { Plântulas }\end{array}$ \\
\hline \multirow{7}{*}{$\begin{array}{l}\text { Rúcula } \\
\text { Cultivada }\end{array}$} & \multicolumn{5}{|c|}{$\%$} \\
\hline & 1 & 5,5 & $98 \mathrm{a}$ & $96 \mathrm{a}$ & $98 \mathrm{a}$ \\
\hline & 2 & 5,0 & $93 \mathrm{a}$ & $82 \mathrm{~b}$ & $89 \mathrm{~b}$ \\
\hline & 3 & 5,4 & $97 \mathrm{a}$ & $94 \mathrm{a}$ & $96 \mathrm{a}$ \\
\hline & 4 & 5,5 & $85 \mathrm{~b}$ & $73 \mathrm{c}$ & $78 \mathrm{c}$ \\
\hline & 5 & 5,0 & $96 \mathrm{a}$ & $95 \mathrm{a}$ & $97 \mathrm{a}$ \\
\hline & $\mathrm{CV}(\%)$ & - & 3,3 & 4,0 & 6,2 \\
\hline \multirow{6}{*}{ Rúcula Gigante } & 6 & 5,5 & $96 \mathrm{a}$ & $96 \mathrm{a}$ & $96 \mathrm{a}$ \\
\hline & 7 & 5,9 & $98 \mathrm{a}$ & $84 \mathrm{~b}$ & $89 \mathrm{~b}$ \\
\hline & 8 & 5,7 & $94 \mathrm{a}$ & $72 \mathrm{c}$ & $75 \mathrm{c}$ \\
\hline & 9 & 5,3 & $94 \mathrm{a}$ & $73 \mathrm{c}$ & $76 \mathrm{c}$ \\
\hline & 10 & 5,3 & $97 \mathrm{a}$ & $95 \mathrm{a}$ & $98 \mathrm{a}$ \\
\hline & $\mathrm{CV}(\%)$ & - & 2,9 & 3,8 & 5,8 \\
\hline
\end{tabular}

* Letras distintas dentro de cada coluna diferem entre si pelo teste de Tukey, a 5\% de probabilidade. 
Verifica-se que para os lotes da Rúcula Cultivada, o teste de germinação destacou o lote 4 como de pior qualidade fisiológica, diferindo estatisticamente dos demais, ou seja, os lotes 1, 2, 3 e 5 foram agrupados num mesmo nível de vigor, não diferindo entre si. Os testes de primeira contagem de germinação e emergência de plântulas em casa de vegetação confirmaram o lote 4 como o de pior qualidade e permitiu destacar os lotes 1 , 3 e 5 como os de maior vigor, não diferindo estatisticamente entre si. O lote 2 se mostrou como intermediário.

Já os resultados dos lotes da Rúcula Gigante não diferiram entre si no teste de germinação. Porém, resultados elevados e semelhantes no teste de germinação não significam necessariamente que todos os lotes possuem alto vigor, uma vez que o teste de germinação é conduzido em condições favoráveis de temperatura, umidade e luminosidade, permitindo ao lote a expressão do potencial máximo para produzir plântulas normais (Marcos Filho, 1999). Nesse sentido, os testes de primeira contagem de germinação e emergência de plântulas em casa de vegetação apresentaram maior sensibilidade, indicando a menor qualidade fisiológica dos lotes 8 e 9 em comparação aos demais lotes.

De maneira semelhante ao teste de primeira contagem de germinação, o teste de emergência de plântulas em casa de vegetação permitiu classificar para as duas cultivares, os lotes de melhor e pior desempenho. O teste de emergência de plântulas constitui um parâmetro indicador da eficiência dos testes para avaliação do potencial fisiológico de lotes de sementes (Marcos Filho, 1999).

Os resultados do teste de condutividade elétrica envolvendo as combinações número de sementes, temperatura e quantidade de água, nos diferentes períodos de embebição, estão apresentados nas Tabelas 2 a 7.

De maneira geral, a análise dos dados mostrou relação direta com os testes de avaliação da qualidade inicial dos lotes de sementes. Para a Rúcula Cultivada, o teste de condutividade elétrica destacou os lotes 1 , 3 e 5 como os de melhor qualidade e o lote 4 como de pior qualidade, corroborando com os dados da emergência em casa de vegetação. Da mesma forma, para a Rúcula Gigante, que apontou os lotes 6 e 10 como os de melhor qualidade e os lotes 8 e 9 como os de pior qualidade. Informações nesse sentido também foram encontradas por Andrade et al. (1995), que verificaram que o teste de condutividade elétrica foi considerado o mais indicado para estimar o vigor de lotes de sementes de cenoura, devido à facilidade de execução, rapidez e objetividade. De maneira semelhante, Dias et al. (1998), em sementes de feijão-de-vagem e quiabo, apontaram o teste de condutividade como o mais eficiente para avaliação do potencial fisiológico dessas sementes.

Com relação ao período de embebição das sementes, verificou-se nas diversas combinações que houve um aumento progressivo das leituras na medida em que se aumentou o período de embebição, corroborando com os dados de Dias et al. (1996). Verificou-se que, de maneira geral, para o período de embebição de 1 hora, em todas as combinações avaliadas, foi possível a estratificação dos lotes quanto ao potencial fisiológico das duas cultivares estudadas. No entanto, essa estratificação só foi considerada consistente após o período de 4 horas de embebição, demonstrando com confiança, a possibilidade de redução no período de embebição das sementes em relação ao período de 24 horas, estabelecido como padrão. Resultados semelhantes foram encontrados por Dias et al. (1996 e 1998), em couve-flor, cebola, cenoura e quiabo; Rodo et al. (1998) em tomate e Torres et al. (1998) em sementes de maxixe, que trabalhando com sementes pequenas de hortaliças, buscando a padronização do teste de condutividade elétrica, observaram significativa lixiviação durante o período de 3 a 4 horas de embebição. Sá (1999), também em sementes de tomate, considerou a possibilidade de redução do tempo de embebição de 24 para 6 horas. Resultados semelhantes foram encontrados por Novembre et al. (2002) em sementes de berinjela com redução do tempo de embebição para 6 horas. Dutra e Vieira (2006), em sementes de abobrinha, constataram que o tempo de embebição pode ser reduzido para 8 horas. Da mesma forma, Oliveira e Novembre (2005) verificaram a possibilidade da redução do tempo de embebição para 6 horas, em sementes de pimentão.

Em relação ao número de sementes, de maneira geral, verificou-se que quando se mantiveram constantes os outros parâmetros avaliados, na medida em que se aumentou o número de sementes, os resultados sofreram pequenos acréscimos nos valores de condutividade, para as duas cultivares avaliadas. Para todas as combinações de número de sementes estudadas ( 25 e 50 sementes), os resultados se mostraram consistentes em diferenciar os lotes com relação ao potencial fisiológico, evidenciando uma relação direta com a emergência em casa de vegetação e primeira contagem de germinação (Tabela 1). Dessa forma, todas as combinações estudadas permitiram estratificar os lotes com estreita relação com a avaliação inicial da qualidade dos mesmos, para as duas cultivares. No entanto, recomenda-se o uso de quatro repetições de 50 sementes, pois o coeficiente de variação com o uso de 25 sementes, para ambas as cultivares, foi maior do que quando se utilizou 50 sementes. 
TABELA 2. Dados médios de condutividade elétrica $\left(\mu \mathrm{S} . \mathrm{cm}^{-1} \cdot \mathrm{g}^{-1}\right)$ utilizando as combinações 25 sementes/25mL, 25 sementes $/ 50 \mathrm{~mL}$ e 25 sementes $/ 75 \mathrm{~mL}$, a $20^{\circ} \mathrm{C}$, de cinco lotes de sementes das cultivares Rúcula Cultivada e Rúcula Gigante, em cada período de embebição.

\begin{tabular}{|c|c|c|c|c|c|c|c|c|c|}
\hline \multirow{2}{*}{ Cultivares } & \multirow{2}{*}{ Lotes } & \multicolumn{8}{|c|}{ Períodos de embebição (h) } \\
\hline & & 1 & 2 & 4 & 6 & 8 & 12 & 18 & 24 \\
\hline & \multicolumn{9}{|c|}{25 sementes $/ 25 \mathrm{~mL} / 20^{\circ} \mathrm{C}$} \\
\hline \multirow{5}{*}{ Rúcula Cultivada } & 1 & $100,6 \mathrm{a}$ & $106,6 \mathrm{a}$ & $112,4 \mathrm{a}$ & $119,3 \mathrm{a}$ & $125,2 \mathrm{a}$ & $127,5 \mathrm{a}$ & $130,7 \mathrm{a}$ & $133,9 \mathrm{a}$ \\
\hline & 2 & $145,4 \mathrm{~b}$ & $149,4 \mathrm{~b}$ & $152,2 \mathrm{~b}$ & $156,6 \mathrm{~b}$ & $157,3 \mathrm{~b}$ & $160,1 \mathrm{~b}$ & $161,5 \mathrm{~b}$ & $162,6 \mathrm{~b}$ \\
\hline & 3 & $104,5 \mathrm{a}$ & $106,7 \mathrm{a}$ & $111,1 \mathrm{a}$ & $113,4 \mathrm{a}$ & 118,9 a & $122,3 \mathrm{a}$ & $128,6 \mathrm{a}$ & $133,8 \mathrm{a}$ \\
\hline & 4 & $143,1 \mathrm{~b}$ & $147,0 \mathrm{~b}$ & $150,9 \mathrm{~b}$ & $154,3 \mathrm{~b}$ & $155,4 \mathrm{~b}$ & $157,9 \mathrm{~b}$ & $158,7 \mathrm{~b}$ & $160,4 b$ \\
\hline & 5 & $103,3 \mathrm{a}$ & $107,2 \mathrm{a}$ & $112,2 \mathrm{a}$ & $117,9 \mathrm{a}$ & $122,9 \mathrm{a}$ & $124,2 \mathrm{a}$ & $129,5 \mathrm{a}$ & $134,0 \mathrm{a}$ \\
\hline \multicolumn{10}{|l|}{ C.V. $(\%)=7,8$} \\
\hline \multirow{5}{*}{ Rúcula Gigante } & 6 & $88,2 \mathrm{a}$ & $92,8 \mathrm{a}$ & $96,1 \mathrm{a}$ & $98,7 \mathrm{a}$ & $104,1 \mathrm{a}$ & $107,8 \mathrm{a}$ & $111,9 \mathrm{a}$ & $129,0 \mathrm{a}$ \\
\hline & 7 & $96,3 \mathrm{ab}$ & $99,5 \mathrm{ab}$ & $107,9 \mathrm{~b}$ & $109,7 \mathrm{~b}$ & $115,5 \mathrm{~b}$ & $119,7 \mathrm{~b}$ & $124,4 \mathrm{~b}$ & $133,4 \mathrm{~b}$ \\
\hline & 8 & $104,0 \mathrm{~b}$ & $108,2 \mathrm{~b}$ & $116,1 \mathrm{c}$ & $118,9 \mathrm{c}$ & $124,2 \mathrm{c}$ & $129,2 \mathrm{c}$ & $133,5 \mathrm{c}$ & $146,6 \mathrm{c}$ \\
\hline & 9 & $103,3 \mathrm{~b}$ & $106,4 \mathrm{~b}$ & $108,6 \mathrm{~b}$ & $110,0 \mathrm{~b}$ & $114,1 \mathrm{~b}$ & $120,4 \mathrm{~b}$ & $126,7 \mathrm{~b}$ & $135,9 \mathrm{~b}$ \\
\hline & 10 & $90,9 \mathrm{a}$ & $94,6 \mathrm{a}$ & $97,4 \mathrm{a}$ & $100,9 \mathrm{a}$ & $105,4 \mathrm{a}$ & $109,4 \mathrm{a}$ & $112,1 \mathrm{a}$ & $121,1 \mathrm{a}$ \\
\hline \multicolumn{10}{|l|}{ C.V. $(\%)=7,5$} \\
\hline & \multicolumn{9}{|c|}{25 sementes $/ 50 \mathrm{~mL} / 20^{\circ} \mathrm{C}$} \\
\hline \multirow{5}{*}{ Rúcula Cultivada } & 1 & 83,6 a & 89,6 a & $95,4 \mathrm{a}$ & $102,3 \mathrm{a}$ & $108,2 \mathrm{a}$ & $110,5 \mathrm{a}$ & $113,7 \mathrm{a}$ & $116,9 \mathrm{a}$ \\
\hline & 2 & $128,4 \mathrm{~b}$ & $132,4 \mathrm{~b}$ & $135,2 \mathrm{~b}$ & $139,6 \mathrm{~b}$ & $140,3 \mathrm{~b}$ & $143,1 \mathrm{~b}$ & $144,5 \mathrm{~b}$ & $145,6 \mathrm{~b}$ \\
\hline & 3 & $87,5 \mathrm{a}$ & $89,7 \mathrm{a}$ & $94,1 \mathrm{a}$ & $96,4 \mathrm{a}$ & 101,9 a & $105,3 \mathrm{a}$ & $111,6 \mathrm{a}$ & $116,8 \mathrm{a}$ \\
\hline & 4 & $126,1 \mathrm{~b}$ & $129,0 \mathrm{~b}$ & $131,9 \mathrm{~b}$ & $133,3 \mathrm{~b}$ & $135,4 \mathrm{~b}$ & $136,9 \mathrm{~b}$ & $140,7 \mathrm{~b}$ & $143,4 b$ \\
\hline & 5 & 86,3 a & $90,2 \mathrm{a}$ & $95,2 \mathrm{a}$ & $100,9 \mathrm{a}$ & $105,9 \mathrm{a}$ & $107,2 \mathrm{a}$ & $112,5 \mathrm{a}$ & $117,0 \mathrm{a}$ \\
\hline \multicolumn{10}{|l|}{ C.V. $(\%)=6,4$} \\
\hline \multirow{5}{*}{ Rúcula Gigante } & 6 & $75,2 \mathrm{a}$ & $79,8 \mathrm{a}$ & $83,1 \mathrm{a}$ & $85,7 \mathrm{a}$ & $91,1 \mathrm{a}$ & $94,8 \mathrm{a}$ & $98,9 \mathrm{a}$ & $106,0 \mathrm{a}$ \\
\hline & 7 & $83,3 \mathrm{ab}$ & $86,5 \mathrm{ab}$ & $94,9 \mathrm{~b}$ & $96,7 \mathrm{~b}$ & $102,5 \mathrm{~b}$ & $106,7 \mathrm{~b}$ & $111,4 \mathrm{~b}$ & $120,4 \mathrm{~b}$ \\
\hline & 8 & $91,0 \mathrm{~b}$ & $95,2 \mathrm{~b}$ & $103,1 \mathrm{c}$ & $105,9 \mathrm{c}$ & $111,2 \mathrm{c}$ & $116,2 \mathrm{c}$ & $120,5 \mathrm{c}$ & $133,6 \mathrm{c}$ \\
\hline & 9 & $90,3 \mathrm{~b}$ & $93,4 \mathrm{~b}$ & $95,6 \mathrm{~b}$ & $97,0 \mathrm{~b}$ & $101,1 \mathrm{~b}$ & $107,4 \mathrm{~b}$ & $112,7 \mathrm{~b}$ & $121,9 \mathrm{~b}$ \\
\hline & 10 & $77,9 \mathrm{a}$ & $81,6 \mathrm{a}$ & $85,4 \mathrm{a}$ & $87,9 \mathrm{a}$ & $92,4 \mathrm{a}$ & $96,4 \mathrm{a}$ & $99,1 \mathrm{a}$ & $108,1 \mathrm{a}$ \\
\hline \multicolumn{10}{|l|}{ C.V. $(\%)=6,2$} \\
\hline \multicolumn{10}{|c|}{25 sementes $/ 75 \mathrm{~mL} / 20^{\circ} \mathrm{C}$} \\
\hline \multirow{5}{*}{ Rúcula Cultivada } & 1 & $74,1 \mathrm{a}$ & $79,3 \mathrm{a}$ & $82,5 \mathrm{a}$ & $85,8 \mathrm{a}$ & $87,4 \mathrm{a}$ & $88,6 \mathrm{a}$ & $80,5 \mathrm{a}$ & $97,2 \mathrm{a}$ \\
\hline & 2 & $99,8 \mathrm{~b}$ & $102,4 \mathrm{~b}$ & $102,9 \mathrm{~b}$ & $104,6 \mathrm{~b}$ & $106,5 \mathrm{~b}$ & $109,5 \mathrm{~b}$ & $111,8 \mathrm{~b}$ & $115,4 \mathrm{~b}$ \\
\hline & 3 & $75,7 \mathrm{a}$ & $79,1 \mathrm{a}$ & $81,2 \mathrm{a}$ & $84,2 \mathrm{a}$ & 86,3 a & $88,4 \mathrm{a}$ & $91,7 \mathrm{a}$ & $96,8 \mathrm{a}$ \\
\hline & 4 & $100,2 \mathrm{~b}$ & $101,4 \mathrm{~b}$ & $103,4 \mathrm{~b}$ & $105,8 \mathrm{~b}$ & $107,1 \mathrm{~b}$ & $111,6 \mathrm{~b}$ & $114,2 \mathrm{~b}$ & $118,7 \mathrm{~b}$ \\
\hline & 5 & $71,9 \mathrm{a}$ & $76,3 \mathrm{a}$ & $80,7 \mathrm{a}$ & $82,7 \mathrm{a}$ & $87,2 \mathrm{a}$ & $89,0 \mathrm{a}$ & $92,6 \mathrm{a}$ & $98,1 \mathrm{a}$ \\
\hline \multicolumn{10}{|l|}{ C.V. $(\%)=6,9$} \\
\hline \multirow{5}{*}{ Rúcula Gigante } & 6 & $69,6 \mathrm{a}$ & $73,6 \mathrm{a}$ & $75,9 \mathrm{a}$ & $84,7 \mathrm{a}$ & $88,2 \mathrm{a}$ & $91,2 \mathrm{a}$ & $94,3 \mathrm{a}$ & $97,4 \mathrm{a}$ \\
\hline & 7 & $70,2 \mathrm{a}$ & $72,5 \mathrm{a}$ & $86,4 \mathrm{~b}$ & $89,9 \mathrm{~b}$ & $93,9 \mathrm{~b}$ & $97,6 \mathrm{~b}$ & $102,5 \mathrm{~b}$ & $107,2 \mathrm{~b}$ \\
\hline & 8 & $84,5 \mathrm{~b}$ & $87,1 \mathrm{~b}$ & $95,3 \mathrm{c}$ & $96,4 \mathrm{c}$ & $98,1 \mathrm{c}$ & $102,4 \mathrm{c}$ & $113,1 \mathrm{c}$ & $120,4 \mathrm{c}$ \\
\hline & 9 & $82,8 \mathrm{~b}$ & $85,6 \mathrm{~b}$ & $84,4 \mathrm{~b}$ & $90,1 \mathrm{~b}$ & $92,5 \mathrm{~b}$ & $97,7 \mathrm{~b}$ & $103,7 \mathrm{~b}$ & $108,3 \mathrm{~b}$ \\
\hline & 10 & $70,4 \mathrm{a}$ & $72,1 \mathrm{a}$ & $76,7 \mathrm{a}$ & $83,2 \mathrm{a}$ & $87,0 \mathrm{a}$ & $90,5 \mathrm{a}$ & $93,8 \mathrm{a}$ & $96,0 \mathrm{a}$ \\
\hline
\end{tabular}

* Letras distintas dentro de cada coluna diferem entre si pelo teste de Tukey, em 5\% de probabilidade. 
TABELA 3. Dados médios de condutividade elétrica $\left(\mu \mathrm{S} . \mathrm{cm}^{-1} \cdot \mathrm{g}^{-1}\right)$ utilizando as combinações 50 sementes/25mL, 50 sementes $/ 50 \mathrm{~mL}$ e 50 sementes $/ 75 \mathrm{~mL}$, a $20^{\circ} \mathrm{C}$, de cinco lotes de sementes das cultivares Rúcula Cultivada e Rúcula Gigante, em cada período de embebição.

\begin{tabular}{|c|c|c|c|c|c|c|c|c|c|}
\hline \multirow{2}{*}{ Cultivares } & \multirow{2}{*}{ Lotes } & \multicolumn{8}{|c|}{ Períodos de embebição (h) } \\
\hline & & 1 & 2 & 4 & 6 & 8 & 12 & 18 & 24 \\
\hline \multicolumn{10}{|c|}{50 sementes $/ 25 \mathrm{~mL} / 20^{\circ} \mathrm{C}$} \\
\hline \multirow{5}{*}{ Rúcula Cultivada } & 1 & $107,4 \mathrm{a}$ & $113,6 \mathrm{a}$ & $118,2 \mathrm{a}$ & $125,1 \mathrm{a}$ & $120,3 \mathrm{a}$ & $131,5 \mathrm{a}$ & $135,4 \mathrm{a}$ & $139,4 \mathrm{a}$ \\
\hline & 2 & $156,5 \mathrm{~b}$ & $159,1 \mathrm{~b}$ & $150,5 \mathrm{~b}$ & $161,2 \mathrm{~b}$ & $162,6 b$ & $162,8 \mathrm{~b}$ & $165,2 \mathrm{~b}$ & $166,1 \mathrm{~b}$ \\
\hline & 3 & $111,6 \mathrm{a}$ & 115,8 a & $119,4 \mathrm{a}$ & $121,4 \mathrm{a}$ & $125,4 \mathrm{a}$ & $117,7 \mathrm{a}$ & $135,6 \mathrm{a}$ & $136,5 \mathrm{a}$ \\
\hline & 4 & $152,7 \mathrm{~b}$ & $153,0 \mathrm{~b}$ & $156,4 \mathrm{~b}$ & $159,0 \mathrm{~b}$ & $161,9 \mathrm{~b}$ & $165,6 \mathrm{~b}$ & $169,2 \mathrm{~b}$ & $170,1 \mathrm{~b}$ \\
\hline & 5 & $104,2 \mathrm{a}$ & $110,3 \mathrm{a}$ & $115,6 \mathrm{a}$ & $120,8 \mathrm{a}$ & $125,4 \mathrm{a}$ & $127,0 \mathrm{a}$ & $131,5 \mathrm{a}$ & $137,3 \mathrm{a}$ \\
\hline \multicolumn{10}{|l|}{ C.V. $(\%)=5,2$} \\
\hline \multirow{5}{*}{ Rúcula Gigante } & 6 & $92,2 \mathrm{a}$ & $97,2 \mathrm{a}$ & $103,8 \mathrm{a}$ & $110,6 \mathrm{a}$ & $116,2 \mathrm{a}$ & $121,8 \mathrm{a}$ & $126,5 \mathrm{a}$ & $133,2 \mathrm{a}$ \\
\hline & 7 & $96,4 \mathrm{a}$ & $103,8 \mathrm{~b}$ & $109,5 \mathrm{~b}$ & $119,2 \mathrm{~b}$ & $127,6 \mathrm{~b}$ & $135,6 \mathrm{~b}$ & $140,8 \mathrm{~b}$ & $149,1 \mathrm{~b}$ \\
\hline & 8 & $112,3 \mathrm{~b}$ & $122,4 \mathrm{c}$ & $126,9 \mathrm{c}$ & $138,8 \mathrm{c}$ & $145,8 \mathrm{c}$ & $150,4 \mathrm{c}$ & $157,0 \mathrm{c}$ & $168,6 \mathrm{c}$ \\
\hline & 9 & $107,8 \mathrm{~b}$ & $108,9 \mathrm{~b}$ & $112,0 \mathrm{~b}$ & $121,0 \mathrm{~b}$ & $124,4 \mathrm{~b}$ & $130,1 \mathrm{~b}$ & $139,6 \mathrm{~b}$ & $157,7 \mathrm{~b}$ \\
\hline & 10 & $93,7 \mathrm{a}$ & $99,4 \mathrm{ab}$ & $94,2 \mathrm{a}$ & $111,7 \mathrm{a}$ & $120,5 \mathrm{a}$ & $125,5 \mathrm{a}$ & $129,7 \mathrm{a}$ & $134,0 \mathrm{a}$ \\
\hline \multicolumn{10}{|l|}{ C.V. $(\%)=4,8$} \\
\hline \multicolumn{10}{|c|}{50 sementes $/ 50 \mathrm{~mL} / 20^{\circ} \mathrm{C}$} \\
\hline \multirow{5}{*}{ Rúcula Cultivada } & 1 & 89,4 a & $95,6 \mathrm{a}$ & $100,2 \mathrm{a}$ & $107,1 \mathrm{a}$ & $102,3 \mathrm{a}$ & 113,5 a & $117,4 \mathrm{a}$ & $121,4 \mathrm{a}$ \\
\hline & 2 & $138,5 \mathrm{~b}$ & $141,1 \mathrm{~b}$ & $132,5 \mathrm{~b}$ & $143,2 \mathrm{~b}$ & $144,6 \mathrm{~b}$ & $144,8 \mathrm{~b}$ & $147,2 \mathrm{~b}$ & $148,1 \mathrm{~b}$ \\
\hline & 3 & 93,6 a & $97,8 \mathrm{a}$ & $101,4 \mathrm{a}$ & $103,4 \mathrm{a}$ & $107,4 \mathrm{a}$ & $99,7 \mathrm{a}$ & $117,6 \mathrm{a}$ & $118,5 \mathrm{a}$ \\
\hline & 4 & $140,7 \mathrm{~b}$ & $145,0 \mathrm{~b}$ & $137,4 \mathrm{~b}$ & $141,0 \mathrm{~b}$ & $142,9 \mathrm{~b}$ & $143,6 \mathrm{~b}$ & $149,2 \mathrm{~b}$ & $147,1 \mathrm{~b}$ \\
\hline & 5 & 86,2 a & $92,3 \mathrm{a}$ & 97,6 a & $102,8 \mathrm{a}$ & $107,4 \mathrm{a}$ & $109,0 \mathrm{a}$ & $113,5 \mathrm{a}$ & $119,3 \mathrm{a}$ \\
\hline \multicolumn{10}{|l|}{ C.V. $(\%)=4,0$} \\
\hline \multirow{5}{*}{ Rúcula Gigante } & 6 & $78,2 \mathrm{a}$ & 83,2 a & 84,8 a & 96,6 a & $102,2 \mathrm{a}$ & $107,8 \mathrm{a}$ & $112,5 \mathrm{a}$ & $119,2 \mathrm{a}$ \\
\hline & 7 & $82,4 \mathrm{a}$ & $89,8 \mathrm{~b}$ & $95,5 \mathrm{~b}$ & $105,2 \mathrm{~b}$ & $113,6 \mathrm{~b}$ & $121,6 \mathrm{~b}$ & $126,8 \mathrm{~b}$ & $135,1 \mathrm{~b}$ \\
\hline & 8 & $102,3 \mathrm{~b}$ & $108,4 \mathrm{c}$ & $112,9 \mathrm{c}$ & $124,8 \mathrm{c}$ & $131,8 \mathrm{c}$ & $136,4 \mathrm{c}$ & $143,0 \mathrm{c}$ & $154,6 \mathrm{c}$ \\
\hline & 9 & $93,8 \mathrm{~b}$ & $94,9 \mathrm{~b}$ & $99,0 \mathrm{~b}$ & $100,0 \mathrm{~b}$ & $118,4 \mathrm{~b}$ & $124,1 \mathrm{~b}$ & $125,6 \mathrm{~b}$ & $133,7 \mathrm{~b}$ \\
\hline & 10 & $79,7 \mathrm{a}$ & $85,4 \mathrm{ab}$ & $80,2 \mathrm{a}$ & $97,7 \mathrm{a}$ & $106,5 \mathrm{a}$ & $111,5 \mathrm{a}$ & $115,7 \mathrm{a}$ & $120,0 \mathrm{a}$ \\
\hline \multicolumn{10}{|l|}{ C.V. $(\%)=4,4$} \\
\hline \multicolumn{10}{|c|}{50 sementes $/ 75 \mathrm{~mL} / 20^{\circ} \mathrm{C}$} \\
\hline \multirow{5}{*}{ Rúcula Cultivada } & 1 & $72,2 \mathrm{a}$ & $81,5 \mathrm{a}$ & $85,2 \mathrm{a}$ & $90,2 \mathrm{a}$ & $93,7 \mathrm{a}$ & 97,5 a & 99,4 a & $103,4 \mathrm{a}$ \\
\hline & 2 & $110,5 \mathrm{~b}$ & $110,7 \mathrm{~b}$ & $113,4 \mathrm{~b}$ & $113,4 b$ & $113,5 \mathrm{~b}$ & $114,2 \mathrm{~b}$ & $114,5 b$ & $114,8 \mathrm{~b}$ \\
\hline & 3 & $78,4 \mathrm{a}$ & $80,4 \mathrm{a}$ & 83,6 a & $85,5 \mathrm{a}$ & $88,4 \mathrm{a}$ & $94,0 \mathrm{a}$ & 96,8 a & $104,6 \mathrm{a}$ \\
\hline & 4 & $105,8 \mathrm{~b}$ & $107,6 \mathrm{~b}$ & $109,7 \mathrm{~b}$ & $110,7 \mathrm{~b}$ & $114,1 \mathrm{~b}$ & $115,7 \mathrm{~b}$ & $117,1 \mathrm{~b}$ & $120,7 \mathrm{~b}$ \\
\hline & 5 & $75,9 \mathrm{a}$ & $78,2 \mathrm{a}$ & $82,3 \mathrm{a}$ & 88,9 a & $91,8 \mathrm{a}$ & $95,6 \mathrm{a}$ & $97,2 \mathrm{a}$ & $101,9 \mathrm{a}$ \\
\hline \multicolumn{10}{|l|}{ C.V. $(\%)=6,1$} \\
\hline \multirow{5}{*}{ Rúcula Gigante } & 6 & $72,8 \mathrm{a}$ & $72,4 \mathrm{a}$ & $82,2 \mathrm{a}$ & $87,1 \mathrm{a}$ & $93,1 \mathrm{a}$ & $98,3 \mathrm{a}$ & $103,2 \mathrm{a}$ & $109,6 \mathrm{a}$ \\
\hline & 7 & $72,4 \mathrm{a}$ & $77,6 \mathrm{a}$ & 83,6 a & 91,5 a & 96,5 a & $102,4 \mathrm{a}$ & $106,4 \mathrm{a}$ & $107,4 \mathrm{a}$ \\
\hline & 8 & $87,6 \mathrm{~b}$ & $93,1 \mathrm{~b}$ & $99,4 \mathrm{~b}$ & $105,2 \mathrm{~b}$ & $113,2 \mathrm{~b}$ & $117,8 \mathrm{~b}$ & $122,5 \mathrm{~b}$ & $126,8 \mathrm{~b}$ \\
\hline & 9 & $83,2 \mathrm{ab}$ & $88,0 \mathrm{ab}$ & $89,1 \mathrm{ab}$ & $101,0 \mathrm{~b}$ & $108,4 \mathrm{~b}$ & $114,6 \mathrm{~b}$ & $118,9 \mathrm{~b}$ & $125,5 \mathrm{~b}$ \\
\hline & 10 & $73,0 \mathrm{a}$ & $76,9 \mathrm{a}$ & $80,5 \mathrm{a}$ & $90,4 \mathrm{a}$ & $95,7 \mathrm{a}$ & $99,2 \mathrm{a}$ & $105,7 \mathrm{a}$ & $110,3 \mathrm{a}$ \\
\hline
\end{tabular}

* Letras distintas dentro de cada coluna diferem entre si pelo teste de Tukey, em 5\% de probabilidade. 
TABELA 4. Dados médios de condutividade elétrica $\left(\mu \mathrm{S} . \mathrm{cm}^{-1} \cdot \mathrm{g}^{-1}\right)$ utilizando as combinações 25 sementes/25mL, 25 sementes/50mL e 25 sementes $/ 75 \mathrm{~mL}$, a $25^{\circ} \mathrm{C}$, de cinco lotes de sementes das cultivares Rúcula Cultivada e Rúcula Gigante, em cada período de embebição.

\begin{tabular}{|c|c|c|c|c|c|c|c|c|c|}
\hline \multirow{2}{*}{ Cultivares } & \multirow{2}{*}{ Lotes } & \multicolumn{8}{|c|}{ Períodos de embebição (h) } \\
\hline & & 1 & 2 & 4 & 6 & 8 & 12 & 18 & 24 \\
\hline \multicolumn{10}{|c|}{25 sementes $/ 25 \mathrm{~mL} / 25^{\circ} \mathrm{C}$} \\
\hline \multirow{5}{*}{ Rúcula Cultivada } & 1 & $110,6 \mathrm{a}$ & $116,6 \mathrm{a}$ & $122,5 \mathrm{a}$ & $130,6 \mathrm{a}$ & $138,4 \mathrm{a}$ & $148,2 \mathrm{a}$ & $158,9 \mathrm{a}$ & $164,4 \mathrm{a}$ \\
\hline & 2 & $157,4 \mathrm{~b}$ & $162,8 \mathrm{~b}$ & $164,9 \mathrm{~b}$ & $165,1 \mathrm{~b}$ & $167,6 \mathrm{~b}$ & $168,4 \mathrm{~b}$ & $175,1 \mathrm{~b}$ & $179,7 \mathrm{~b}$ \\
\hline & 3 & 113,6 a & $117,7 \mathrm{a}$ & $121,1 \mathrm{a}$ & $138,2 \mathrm{a}$ & $138,0 \mathrm{a}$ & $146,4 \mathrm{a}$ & $155,0 \mathrm{a}$ & $166,5 \mathrm{a}$ \\
\hline & 4 & $169,9 \mathrm{c}$ & $174,4 \mathrm{c}$ & $178,2 \mathrm{c}$ & $183,7 \mathrm{c}$ & $188,1 \mathrm{c}$ & $193,9 \mathrm{c}$ & $175,6 \mathrm{c}$ & $213,2 \mathrm{c}$ \\
\hline & 5 & $107,8 \mathrm{a}$ & $112,6 \mathrm{a}$ & $121,0 \mathrm{a}$ & $138,6 \mathrm{a}$ & $136,9 \mathrm{a}$ & $143,1 \mathrm{a}$ & $154,9 \mathrm{a}$ & $163,4 \mathrm{a}$ \\
\hline \multicolumn{10}{|l|}{ C.V. $(\%)=9,6$} \\
\hline \multirow{5}{*}{ Rúcula Gigante } & 6 & $96,1 \mathrm{a}$ & $100,6 \mathrm{a}$ & $110,2 \mathrm{a}$ & $121,4 \mathrm{a}$ & $127,6 \mathrm{a}$ & $139,0 \mathrm{a}$ & $154,2 \mathrm{a}$ & $161,5 \mathrm{a}$ \\
\hline & 7 & 98,6 a & $106,5 \mathrm{~b}$ & $124,4 \mathrm{~b}$ & $130,3 \mathrm{~b}$ & $140,4 \mathrm{~b}$ & $149,4 \mathrm{~b}$ & $161,3 \mathrm{~b}$ & $164,6 \mathrm{~b}$ \\
\hline & 8 & $119,2 \mathrm{~b}$ & $120,3 \mathrm{c}$ & $143,2 \mathrm{c}$ & $149,0 \mathrm{c}$ & $157,2 \mathrm{c}$ & $163,7 \mathrm{c}$ & $177,3 \mathrm{c}$ & $188,8 \mathrm{c}$ \\
\hline & 9 & $114,6 \mathrm{~b}$ & $119,2 \mathrm{c}$ & $141,4 \mathrm{c}$ & $144,9 \mathrm{c}$ & $154,9 \mathrm{c}$ & $166,6 \mathrm{c}$ & $182,6 \mathrm{c}$ & $194,6 \mathrm{c}$ \\
\hline & 10 & 96,7 a & $101,8 \mathrm{a}$ & $110,3 \mathrm{a}$ & $121,5 \mathrm{a}$ & $126,7 \mathrm{a}$ & $138,8 \mathrm{a}$ & $157,2 \mathrm{a}$ & $162,9 \mathrm{a}$ \\
\hline \multicolumn{10}{|l|}{ C.V. $(\%)=9,4$} \\
\hline \multicolumn{10}{|c|}{25 sementes $/ 50 \mathrm{~mL} / 25^{\circ} \mathrm{C}$} \\
\hline \multirow{5}{*}{ Rúcula Cultivada } & 1 & $92,6 \mathrm{a}$ & $98,6 \mathrm{a}$ & $104,5 \mathrm{a}$ & $112,6 \mathrm{a}$ & $120,4 \mathrm{a}$ & $130,2 \mathrm{a}$ & $140,9 \mathrm{a}$ & $146,4 \mathrm{a}$ \\
\hline & 2 & $139,4 \mathrm{~b}$ & $144,8 \mathrm{~b}$ & $146,9 \mathrm{~b}$ & $147,1 \mathrm{~b}$ & $149,6 \mathrm{~b}$ & $150,4 \mathrm{~b}$ & $157,1 \mathrm{~b}$ & $161,7 \mathrm{~b}$ \\
\hline & 3 & $95,6 \mathrm{a}$ & $99,7 \mathrm{a}$ & $103,1 \mathrm{a}$ & $120,2 \mathrm{a}$ & $120,0 \mathrm{a}$ & $128,4 \mathrm{a}$ & $137,0 \mathrm{a}$ & $148,5 \mathrm{a}$ \\
\hline & 4 & $151,9 \mathrm{c}$ & $156,4 \mathrm{c}$ & $160,2 \mathrm{c}$ & $165,7 \mathrm{c}$ & $170,1 \mathrm{c}$ & $175,9 \mathrm{c}$ & $157,6 \mathrm{c}$ & $195,2 \mathrm{c}$ \\
\hline & 5 & $89,8 \mathrm{a}$ & 94,6 a & $103,0 \mathrm{a}$ & $120,6 \mathrm{a}$ & $118,9 \mathrm{a}$ & $125,1 \mathrm{a}$ & $136,9 \mathrm{a}$ & $145,4 \mathrm{a}$ \\
\hline \multicolumn{10}{|l|}{ C.V. $(\%)=7,0$} \\
\hline \multirow{5}{*}{ Rúcula Gigante } & 6 & $82,1 \mathrm{a}$ & $86,6 \mathrm{a}$ & $96,2 \mathrm{a}$ & $107,4 \mathrm{a}$ & $113,6 \mathrm{a}$ & $125,0 \mathrm{a}$ & $140,2 \mathrm{a}$ & $147,5 \mathrm{a}$ \\
\hline & 7 & 84,6 a & $92,5 \mathrm{~b}$ & $110,4 \mathrm{~b}$ & $116,3 \mathrm{~b}$ & $126,4 \mathrm{~b}$ & $135,4 \mathrm{~b}$ & $147,3 \mathrm{~b}$ & $150,6 b$ \\
\hline & 8 & $105,2 \mathrm{~b}$ & $106,3 \mathrm{c}$ & $129,2 \mathrm{c}$ & $135,0 \mathrm{c}$ & $143,2 \mathrm{c}$ & $149,7 \mathrm{c}$ & $163,3 \mathrm{c}$ & $174,8 \mathrm{c}$ \\
\hline & 9 & $100,6 \mathrm{~b}$ & $105,2 \mathrm{c}$ & $127,4 \mathrm{c}$ & $130,9 \mathrm{c}$ & $140,9 \mathrm{c}$ & $152,6 \mathrm{c}$ & $168,6 \mathrm{c}$ & $180,6 \mathrm{c}$ \\
\hline & 10 & $82,7 \mathrm{a}$ & $87,8 \mathrm{a}$ & $96,3 \mathrm{a}$ & $107,5 \mathrm{a}$ & $112,7 \mathrm{a}$ & $124,8 \mathrm{a}$ & $143,2 \mathrm{a}$ & $148,9 \mathrm{a}$ \\
\hline \multicolumn{10}{|l|}{ C.V. $(\%)=7,1$} \\
\hline \multicolumn{10}{|c|}{25 sementes $/ 75 \mathrm{~mL} / 25^{\circ} \mathrm{C}$} \\
\hline \multirow{5}{*}{ Rúcula Cultivada } & 1 & $75,5 \mathrm{a}$ & $78,9 \mathrm{a}$ & $85,4 \mathrm{a}$ & $95,6 \mathrm{a}$ & $102,2 \mathrm{a}$ & $106,1 \mathrm{a}$ & $110,4 \mathrm{a}$ & $117,4 \mathrm{a}$ \\
\hline & 2 & $109,6 \mathrm{~b}$ & $112,3 \mathrm{~b}$ & $113,2 \mathrm{~b}$ & $116,4 b$ & $117,3 \mathrm{~b}$ & $119,8 \mathrm{~b}$ & $122,7 \mathrm{~b}$ & $123,6 \mathrm{~b}$ \\
\hline & 3 & $83,1 \mathrm{a}$ & $85,2 \mathrm{a}$ & $88,7 \mathrm{a}$ & $94,7 \mathrm{a}$ & $99,8 \mathrm{a}$ & $100,4 \mathrm{a}$ & $109,6 \mathrm{a}$ & $114,8 \mathrm{a}$ \\
\hline & 4 & $115,0 \mathrm{~b}$ & $122,4 \mathrm{c}$ & $126,6 \mathrm{c}$ & $133,8 \mathrm{c}$ & $137,1 \mathrm{c}$ & $142,7 \mathrm{c}$ & $146,2 \mathrm{c}$ & $149,7 \mathrm{c}$ \\
\hline & 5 & $75,3 \mathrm{a}$ & $79,1 \mathrm{a}$ & $84,5 \mathrm{a}$ & $93,9 \mathrm{a}$ & $98,0 \mathrm{a}$ & $106,5 \mathrm{a}$ & $113,8 \mathrm{a}$ & $116,3 \mathrm{a}$ \\
\hline \multicolumn{10}{|l|}{ C.V. $(\%)=8,6$} \\
\hline \multirow{5}{*}{ Rúcula Gigante } & 6 & $70,7 \mathrm{a}$ & $75,6 \mathrm{a}$ & $81,5 \mathrm{a}$ & $90,4 \mathrm{a}$ & $100,4 \mathrm{a}$ & $106,7 \mathrm{a}$ & $113,2 \mathrm{a}$ & $120,3 \mathrm{a}$ \\
\hline & 7 & $73,2 \mathrm{a}$ & $79,8 \mathrm{a}$ & $96,9 \mathrm{~b}$ & $98,6 \mathrm{~b}$ & $109,5 \mathrm{~b}$ & $115,4 \mathrm{~b}$ & $122,4 \mathrm{~b}$ & $126,5 \mathrm{~b}$ \\
\hline & 8 & 89,6 b & $96,3 \mathrm{~b}$ & $110,7 \mathrm{c}$ & $114,7 \mathrm{c}$ & $121,7 \mathrm{c}$ & $128,9 \mathrm{c}$ & $132,8 \mathrm{c}$ & $139,8 \mathrm{c}$ \\
\hline & 9 & $85,4 \mathrm{~b}$ & $91,4 \mathrm{~b}$ & $109,6 \mathrm{c}$ & $119,5 \mathrm{c}$ & $122,4 \mathrm{c}$ & $127,3 \mathrm{c}$ & $135,6 \mathrm{c}$ & $140,7 \mathrm{c}$ \\
\hline & 10 & $72,1 \mathrm{a}$ & $77,5 \mathrm{a}$ & $83,0 \mathrm{a}$ & $92,3 \mathrm{a}$ & $101,8 \mathrm{a}$ & $109,1 \mathrm{a}$ & $115,9 \mathrm{a}$ & $122,1 \mathrm{a}$ \\
\hline
\end{tabular}

* Letras distintas dentro de cada coluna diferem entre si pelo teste de Tukey, em 5\% de probabilidade. 
TABELA 5. Dados médios de condutividade elétrica $\left(\mu \mathrm{S} . \mathrm{cm}^{-1} \cdot \mathrm{g}^{-1}\right)$ utilizando as combinações 50 sementes/25mL, 50 sementes $/ 50 \mathrm{~mL}$ e 50 sementes $/ 75 \mathrm{~mL}$, a $25^{\circ} \mathrm{C}$, de cinco lotes de sementes das cultivares Rúcula Cultivada e Rúcula Gigante, em cada período de embebição.

\begin{tabular}{|c|c|c|c|c|c|c|c|c|c|}
\hline \multirow{2}{*}{ Cultivares } & \multirow{2}{*}{$\begin{array}{l}\text { Lote } \\
\mathrm{s}\end{array}$} & \multicolumn{8}{|c|}{ Períodos de embebição (h) } \\
\hline & & 1 & 2 & 4 & 6 & 8 & 12 & 18 & 24 \\
\hline \multicolumn{10}{|c|}{50 sementes $/ 25 \mathrm{~mL} / 25^{\circ} \mathrm{C}$} \\
\hline \multirow{5}{*}{$\begin{array}{l}\text { Rúcula } \\
\text { Cultivada }\end{array}$} & 1 & $113,5 \mathrm{a}$ & $120,2 \mathrm{a}$ & $129,2 \mathrm{a}$ & $140,4 \mathrm{a}$ & $149,0 \mathrm{a}$ & $154,7 \mathrm{a}$ & $161,1 \mathrm{a}$ & $167,5 \mathrm{a}$ \\
\hline & 2 & $160,3 \mathrm{~b}$ & $164,7 \mathrm{~b}$ & $149,4 \mathrm{~b}$ & $172,6 \mathrm{~b}$ & $173,8 \mathrm{~b}$ & $177,2 \mathrm{~b}$ & $183,3 \mathrm{~b}$ & $195,0 \mathrm{~b}$ \\
\hline & 3 & $117,7 \mathrm{a}$ & $121,6 \mathrm{a}$ & $130,7 \mathrm{a}$ & 137,9 a & $144,6 \mathrm{a}$ & $153,2 \mathrm{a}$ & $164,0 \mathrm{a}$ & $168,6 \mathrm{a}$ \\
\hline & 4 & $171,9 \mathrm{c}$ & $181,1 \mathrm{c}$ & $187,8 \mathrm{c}$ & $199,7 \mathrm{c}$ & $209,3 \mathrm{c}$ & $213,5 \mathrm{c}$ & $222,6 \mathrm{c}$ & $235,9 \mathrm{c}$ \\
\hline & 5 & $107,0 \mathrm{a}$ & $114,5 \mathrm{a}$ & $122,4 \mathrm{a}$ & $137,0 \mathrm{a}$ & $145,4 \mathrm{a}$ & $151,2 \mathrm{a}$ & $160,2 \mathrm{a}$ & $165,9 \mathrm{a}$ \\
\hline \multicolumn{10}{|c|}{ C.V. $(\%)=6,6$} \\
\hline \multirow{5}{*}{$\begin{array}{l}\text { Rúcula } \\
\text { Gigante }\end{array}$} & 6 & $103,0 \mathrm{a}$ & $107,3 \mathrm{a}$ & $117,3 \mathrm{a}$ & $129,1 \mathrm{a}$ & $137,3 \mathrm{a}$ & $145,2 \mathrm{a}$ & $154,4 \mathrm{a}$ & $163,6 \mathrm{a}$ \\
\hline & 7 & $104,5 \mathrm{a}$ & $113,4 b$ & $130,6 \mathrm{~b}$ & $142,3 \mathrm{~b}$ & $156,6 \mathrm{~b}$ & $163,6 b$ & $169,8 \mathrm{~b}$ & $175,2 \mathrm{~b}$ \\
\hline & 8 & $125,5 \mathrm{~b}$ & $127,7 \mathrm{c}$ & $142,4 \mathrm{c}$ & $162,8 \mathrm{c}$ & $165,2 \mathrm{c}$ & $170,7 \mathrm{c}$ & $181,2 \mathrm{c}$ & $194,5 \mathrm{c}$ \\
\hline & 9 & $121,3 \mathrm{~b}$ & $127,5 \mathrm{c}$ & $131,0 \mathrm{c}$ & $158,9 \mathrm{c}$ & $162,4 \mathrm{c}$ & $172,4 \mathrm{c}$ & $184,1 \mathrm{c}$ & $197,2 \mathrm{c}$ \\
\hline & 10 & $102,4 \mathrm{a}$ & $107,1 \mathrm{a}$ & $117,8 \mathrm{a}$ & $131,0 \mathrm{a}$ & $140,1 \mathrm{a}$ & $154,5 \mathrm{a}$ & $155,2 \mathrm{a}$ & $166,9 \mathrm{a}$ \\
\hline \multicolumn{10}{|l|}{ C.V. $(\%)=6,1$} \\
\hline \multicolumn{10}{|c|}{50 sementes $/ 50 \mathrm{~mL} / 25^{\circ} \mathrm{C}$} \\
\hline \multirow{5}{*}{$\begin{array}{l}\text { Rúcula } \\
\text { Cultivada }\end{array}$} & 1 & $94,5 \mathrm{a}$ & $101,2 \mathrm{a}$ & $110,2 \mathrm{a}$ & $121,4 \mathrm{a}$ & $130,0 \mathrm{a}$ & $135,7 \mathrm{a}$ & $142,1 \mathrm{a}$ & $148,5 \mathrm{a}$ \\
\hline & 2 & $141,3 \mathrm{~b}$ & $145,7 \mathrm{~b}$ & $130,4 \mathrm{~b}$ & $153,6 \mathrm{~b}$ & $154,8 \mathrm{~b}$ & $158,2 \mathrm{~b}$ & $164,3 \mathrm{~b}$ & $176,0 \mathrm{~b}$ \\
\hline & 3 & 98,7 a & $102,6 \mathrm{a}$ & $111,7 \mathrm{a}$ & 118,9 a & $125,6 \mathrm{a}$ & $134,2 \mathrm{a}$ & $145,0 \mathrm{a}$ & $149,6 \mathrm{a}$ \\
\hline & 4 & $152,9 \mathrm{c}$ & $162,1 \mathrm{c}$ & $168,8 \mathrm{c}$ & $180,7 \mathrm{c}$ & $190,3 \mathrm{c}$ & $194,5 \mathrm{c}$ & $203,6 \mathrm{c}$ & $216,9 \mathrm{c}$ \\
\hline & 5 & 88,0 a & $95,5 \mathrm{a}$ & $103,4 \mathrm{a}$ & $118,0 \mathrm{a}$ & $126,4 \mathrm{a}$ & $132,2 \mathrm{a}$ & $141,2 \mathrm{a}$ & $146,9 \mathrm{a}$ \\
\hline \multicolumn{10}{|c|}{ C.V. $(\%)=3,4$} \\
\hline \multirow{5}{*}{$\begin{array}{l}\text { Rúcula } \\
\text { Gigante }\end{array}$} & 6 & 88,0 a & 92,3 a & $102,3 \mathrm{a}$ & $114,1 \mathrm{a}$ & $122,3 \mathrm{a}$ & $130,2 \mathrm{a}$ & $139,4 \mathrm{a}$ & $148,6 \mathrm{a}$ \\
\hline & 7 & 89,5 a & $98,4 \mathrm{~b}$ & $115,6 \mathrm{~b}$ & $127,3 \mathrm{~b}$ & $141,6 \mathrm{~b}$ & $148,6 \mathrm{~b}$ & $154,8 \mathrm{~b}$ & $160,2 \mathrm{~b}$ \\
\hline & 8 & $110,5 \mathrm{~b}$ & $112,7 \mathrm{c}$ & $127,4 \mathrm{c}$ & $147,8 \mathrm{c}$ & $150,2 \mathrm{c}$ & $155,7 \mathrm{c}$ & $166,2 \mathrm{c}$ & $179,5 \mathrm{c}$ \\
\hline & 9 & $106,3 \mathrm{~b}$ & $112,5 \mathrm{c}$ & $116,0 \mathrm{c}$ & $143,9 \mathrm{c}$ & $147,4 \mathrm{c}$ & $157,4 \mathrm{c}$ & $169,1 \mathrm{c}$ & $182,2 \mathrm{c}$ \\
\hline & 10 & 87,4 a & $92,1 \mathrm{a}$ & $102,8 \mathrm{a}$ & $116,0 \mathrm{a}$ & $125,1 \mathrm{a}$ & $139,5 \mathrm{a}$ & $140,2 \mathrm{a}$ & $151,9 \mathrm{a}$ \\
\hline \multicolumn{10}{|c|}{ C.V. $(\%)=3,7$} \\
\hline \multicolumn{10}{|c|}{50 sementes $/ 75 \mathrm{~mL} / 25^{\circ} \mathrm{C}$} \\
\hline \multirow{5}{*}{$\begin{array}{l}\text { Rúcula } \\
\text { Cultivada }\end{array}$} & 1 & 80,3 a & $85,4 \mathrm{a}$ & 90,5 a & $101,2 \mathrm{a}$ & $106,1 \mathrm{a}$ & $109,6 \mathrm{a}$ & $112,5 \mathrm{a}$ & $118,9 \mathrm{a}$ \\
\hline & 2 & $110,4 \mathrm{~b}$ & $112,5 \mathrm{~b}$ & $115,6 \mathrm{~b}$ & $122,4 \mathrm{~b}$ & $124,5 \mathrm{~b}$ & $123,4 \mathrm{~b}$ & $126,4 \mathrm{~b}$ & $128,4 \mathrm{~b}$ \\
\hline & 3 & $88,9 \mathrm{ab}$ & $92,8 \mathrm{ab}$ & $93,1 \mathrm{a}$ & $103,8 \mathrm{a}$ & $108,9 \mathrm{a}$ & $111,7 \mathrm{a}$ & $116,9 \mathrm{a}$ & $121,7 \mathrm{a}$ \\
\hline & 4 & $114,7 \mathrm{~b}$ & $115,7 \mathrm{~b}$ & $125,2 \mathrm{c}$ & $135,7 \mathrm{c}$ & $145,7 \mathrm{c}$ & $151,2 \mathrm{c}$ & $157,1 \mathrm{c}$ & $161,2 \mathrm{c}$ \\
\hline & 5 & $81,3 \mathrm{a}$ & $84,2 \mathrm{a}$ & 88,9 a & 97,9 a & $105,2 \mathrm{a}$ & $109,3 \mathrm{a}$ & $116,0 \mathrm{a}$ & $120,3 \mathrm{a}$ \\
\hline \multicolumn{10}{|l|}{ C.V. $(\%)=6,8$} \\
\hline \multirow{5}{*}{$\begin{array}{l}\text { Rúcula } \\
\text { Gigante }\end{array}$} & 6 & $72,1 \mathrm{a}$ & $80,1 \mathrm{a}$ & 84,9 a & 94,5 a & $101,3 \mathrm{a}$ & $106,2 \mathrm{a}$ & $112,1 \mathrm{a}$ & $115,2 \mathrm{a}$ \\
\hline & 7 & $75,4 \mathrm{a}$ & 83,5 a & $92,1 \mathrm{~b}$ & $102,3 \mathrm{~b}$ & $111,5 \mathrm{~b}$ & $118,4 \mathrm{~b}$ & $123,8 \mathrm{~b}$ & $129,7 \mathrm{~b}$ \\
\hline & 8 & $93,6 \mathrm{~b}$ & $98,7 \mathrm{c}$ & $105,3 \mathrm{c}$ & $121,7 \mathrm{c}$ & $131,7 \mathrm{c}$ & $137,9 \mathrm{c}$ & $147,2 \mathrm{c}$ & $162,3 \mathrm{c}$ \\
\hline & 9 & $88,2 \mathrm{~b}$ & $95,8 \mathrm{c}$ & $103,4 \mathrm{c}$ & $116,0 \mathrm{c}$ & $127,6 \mathrm{c}$ & $134,0 \mathrm{c}$ & $141,6 \mathrm{c}$ & $160,4 \mathrm{c}$ \\
\hline & 10 & 73,9 a & 78,0 a & $82,7 \mathrm{a}$ & $90,8 \mathrm{a}$ & $98,1 \mathrm{a}$ & $105,2 \mathrm{a}$ & $110,4 \mathrm{a}$ & $112,1 \mathrm{a}$ \\
\hline
\end{tabular}

* Letras distintas dentro de cada coluna diferem entre si pelo teste de Tukey, em 5\% de probabilidade. 


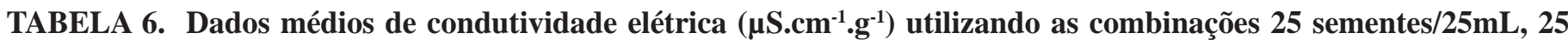
sementes/50mL e 25 sementes $/ 75 \mathrm{~mL}$, a $30^{\circ} \mathrm{C}$, de cinco lotes de sementes das cultivares Rúcula Cultivada e Rúcula Gigante, em cada período de embebição.

\begin{tabular}{|c|c|c|c|c|c|c|c|c|c|}
\hline \multirow{2}{*}{ Cultivares } & \multirow{2}{*}{ Lotes } & \multicolumn{8}{|c|}{ Períodos de embebição (h) } \\
\hline & & 1 & 2 & 4 & 6 & 8 & 12 & 18 & 24 \\
\hline \multicolumn{10}{|c|}{25 sementes $/ 25 \mathrm{~mL} / 30^{\circ} \mathrm{C}$} \\
\hline \multirow{5}{*}{ Rúcula Cultivada } & 1 & 116,8 a & $121,2 \mathrm{a}$ & $130,8 \mathrm{a}$ & $139,1 \mathrm{a}$ & $149,5 \mathrm{a}$ & 156,0 a & $159,2 \mathrm{a}$ & $164,2 \mathrm{a}$ \\
\hline & 2 & $165,0 \mathrm{~b}$ & $166,8 \mathrm{~b}$ & $169,2 \mathrm{~b}$ & $173,6 \mathrm{~b}$ & $177,5 \mathrm{~b}$ & $178,8 \mathrm{~b}$ & $180,6 \mathrm{~b}$ & $189,3 \mathrm{~b}$ \\
\hline & 3 & 116,6 a & $122,6 \mathrm{a}$ & $127,0 \mathrm{a}$ & $141,9 \mathrm{a}$ & $149,7 \mathrm{a}$ & $153,2 \mathrm{a}$ & 156,9 a & $164,2 \mathrm{a}$ \\
\hline & 4 & $168,3 \mathrm{c}$ & $171,8 \mathrm{c}$ & $184,4 \mathrm{c}$ & $199,6 \mathrm{c}$ & $203,1 \mathrm{c}$ & 204,9 c & $208,2 \mathrm{c}$ & $209,6 \mathrm{c}$ \\
\hline & 5 & $120,4 \mathrm{a}$ & $124,8 \mathrm{a}$ & $132,3 \mathrm{a}$ & $137,9 \mathrm{a}$ & $149,2 \mathrm{a}$ & $157,4 \mathrm{a}$ & $159,1 \mathrm{a}$ & $161,5 \mathrm{a}$ \\
\hline \multicolumn{10}{|l|}{ C.V. $(\%)=9,4$} \\
\hline \multirow{5}{*}{ Rúcula Gigante } & 6 & $101,6 \mathrm{a}$ & $108,1 \mathrm{a}$ & $127,2 \mathrm{a}$ & $132,2 \mathrm{a}$ & $147,1 \mathrm{a}$ & $158,1 \mathrm{a}$ & $174,3 \mathrm{a}$ & $175,2 \mathrm{a}$ \\
\hline & 7 & $105,1 \mathrm{a}$ & $113,4 \mathrm{a}$ & $136,4 \mathrm{~b}$ & $145,5 \mathrm{~b}$ & $158,8 \mathrm{~b}$ & $169,8 \mathrm{~b}$ & $176,4 \mathrm{~b}$ & $182,0 \mathrm{~b}$ \\
\hline & 8 & $124,8 \mathrm{~b}$ & $134,9 \mathrm{~b}$ & $147,0 \mathrm{c}$ & $155,3 \mathrm{c}$ & $174,1 \mathrm{c}$ & $185,6 \mathrm{c}$ & $194,2 \mathrm{c}$ & $205,3 \mathrm{c}$ \\
\hline & 9 & $121,3 \mathrm{~b}$ & $130,7 \mathrm{~b}$ & $148,8 \mathrm{c}$ & $156,6 \mathrm{c}$ & $169,5 \mathrm{c}$ & $183,2 \mathrm{c}$ & $190,3 \mathrm{c}$ & $202,5 \mathrm{c}$ \\
\hline & 10 & $100,4 \mathrm{a}$ & $109,0 \mathrm{a}$ & 126,9 a & $132,8 \mathrm{a}$ & $150,2 \mathrm{a}$ & $159,0 \mathrm{a}$ & $172,1 \mathrm{a}$ & $175,9 \mathrm{a}$ \\
\hline \multicolumn{10}{|l|}{ C.V. $(\%)=9,7$} \\
\hline \multicolumn{10}{|c|}{25 sementes $/ 50 \mathrm{~mL} / 30^{\circ} \mathrm{C}$} \\
\hline \multirow{5}{*}{ Rúcula Cultivada } & 1 & $97,8 \mathrm{a}$ & $102,2 \mathrm{a}$ & $111,8 \mathrm{a}$ & $120,1 \mathrm{a}$ & $130,5 \mathrm{a}$ & $137,0 \mathrm{a}$ & $140,2 \mathrm{a}$ & $145,2 \mathrm{a}$ \\
\hline & 2 & $146,0 \mathrm{~b}$ & $147,8 \mathrm{~b}$ & $150,2 \mathrm{~b}$ & $154,6 \mathrm{~b}$ & $158,5 \mathrm{~b}$ & $159,8 \mathrm{~b}$ & $161,6 \mathrm{~b}$ & $170,3 \mathrm{~b}$ \\
\hline & 3 & 97,6 a & 103,6 a & $108,0 \mathrm{a}$ & $122,9 \mathrm{a}$ & $130,7 \mathrm{a}$ & $134,2 \mathrm{a}$ & 137,9 a & $145,2 \mathrm{a}$ \\
\hline & 4 & $149,3 \mathrm{c}$ & $152,8 \mathrm{c}$ & $165,4 \mathrm{c}$ & $180,6 \mathrm{c}$ & $184,1 \mathrm{c}$ & $185,9 \mathrm{c}$ & $189,2 \mathrm{c}$ & $190,6 \mathrm{c}$ \\
\hline & 5 & $101,4 \mathrm{a}$ & $105,8 \mathrm{a}$ & $113,3 \mathrm{a}$ & $118,9 \mathrm{a}$ & $130,2 \mathrm{a}$ & $138,4 \mathrm{a}$ & $140,1 \mathrm{a}$ & $142,5 \mathrm{a}$ \\
\hline \multicolumn{10}{|l|}{ C.V. $(\%)=6,5$} \\
\hline \multirow{5}{*}{ Rúcula Gigante } & 6 & 86,6 a & $93,1 \mathrm{a}$ & $112,2 \mathrm{a}$ & $117,2 \mathrm{a}$ & $132,1 \mathrm{a}$ & $143,1 \mathrm{a}$ & 159,3 a & $160,2 \mathrm{a}$ \\
\hline & 7 & $90,1 \mathrm{a}$ & $98,4 \mathrm{a}$ & $121,4 \mathrm{~b}$ & $130,5 \mathrm{~b}$ & $143,8 \mathrm{~b}$ & $154,8 \mathrm{~b}$ & $161,4 \mathrm{~b}$ & $167,0 \mathrm{~b}$ \\
\hline & 8 & $109,8 \mathrm{~b}$ & $119,9 \mathrm{~b}$ & $132,0 \mathrm{c}$ & $140,3 \mathrm{c}$ & $159,1 \mathrm{c}$ & $170,6 \mathrm{c}$ & $179,2 \mathrm{c}$ & $190,3 \mathrm{c}$ \\
\hline & 9 & $106,3 \mathrm{~b}$ & $115,7 \mathrm{~b}$ & $133,8 \mathrm{c}$ & $141,6 \mathrm{c}$ & $154,5 \mathrm{c}$ & $168,2 \mathrm{c}$ & $175,3 \mathrm{c}$ & $187,5 \mathrm{c}$ \\
\hline & 10 & $85,4 \mathrm{a}$ & $94,0 \mathrm{a}$ & $111,9 \mathrm{a}$ & $117,8 \mathrm{a}$ & $135,2 \mathrm{a}$ & $144,0 \mathrm{a}$ & $157,1 \mathrm{a}$ & $160,9 \mathrm{a}$ \\
\hline \multicolumn{10}{|l|}{ C.V. $(\%)=6,4$} \\
\hline \multicolumn{10}{|c|}{25 sementes $/ 75 \mathrm{~mL} / 30^{\circ} \mathrm{C}$} \\
\hline \multirow{5}{*}{ Rúcula Cultivada } & 1 & $80,4 \mathrm{a}$ & 85,6 a & $91,6 \mathrm{a}$ & $100,8 \mathrm{a}$ & $106,3 \mathrm{a}$ & $111,3 \mathrm{a}$ & $112,5 \mathrm{a}$ & $123,1 \mathrm{a}$ \\
\hline & 2 & $112,3 \mathrm{~b}$ & $112,3 \mathrm{~b}$ & $115,4 \mathrm{~b}$ & $119,4 b$ & $120,7 \mathrm{~b}$ & $121,4 \mathrm{~b}$ & $128,7 \mathrm{~b}$ & $135,4 \mathrm{~b}$ \\
\hline & 3 & $83,7 \mathrm{a}$ & 90,7 a & $92,8 \mathrm{a}$ & $100,3 \mathrm{a}$ & $104,1 \mathrm{a}$ & $107,6 \mathrm{a}$ & 113,3 a & $122,6 \mathrm{a}$ \\
\hline & 4 & $118,9 \mathrm{~b}$ & $120,4 \mathrm{~b}$ & $125,0 \mathrm{c}$ & $138,6 \mathrm{c}$ & $138,9 \mathrm{c}$ & $140,2 \mathrm{c}$ & $145,0 \mathrm{c}$ & $149,7 \mathrm{c}$ \\
\hline & 5 & $78,0 \mathrm{a}$ & $81,5 \mathrm{a}$ & $87,2 \mathrm{a}$ & $95,4 \mathrm{a}$ & $101,8 \mathrm{a}$ & $109,8 \mathrm{a}$ & $114,6 \mathrm{a}$ & $121,8 \mathrm{a}$ \\
\hline \multicolumn{10}{|l|}{ C.V. $(\%)=8,9$} \\
\hline \multirow{5}{*}{ Rúcula Gigante } & 6 & $73,5 \mathrm{a}$ & 78,9 a & 84,5 a & $94,6 \mathrm{a}$ & $104,8 \mathrm{a}$ & $113,2 \mathrm{a}$ & $117,4 \mathrm{a}$ & $119,6 \mathrm{a}$ \\
\hline & 7 & $75,8 \mathrm{a}$ & $81,2 \mathrm{a}$ & $90,7 \mathrm{~b}$ & $104,7 \mathrm{~b}$ & $112,2 \mathrm{~b}$ & $117,8 \mathrm{~b}$ & $123,6 \mathrm{~b}$ & $126,4 \mathrm{~b}$ \\
\hline & 8 & $88,4 \mathrm{~b}$ & $95,6 \mathrm{~b}$ & $108,4 \mathrm{c}$ & $119,2 \mathrm{c}$ & $128,3 \mathrm{c}$ & $139,5 \mathrm{c}$ & $153,2 \mathrm{c}$ & $165,7 \mathrm{c}$ \\
\hline & 9 & $87,1 \mathrm{~b}$ & $93,2 \mathrm{~b}$ & $103,6 \mathrm{c}$ & $113,4 \mathrm{c}$ & $123,7 \mathrm{c}$ & $135,2 \mathrm{c}$ & $143,9 \mathrm{c}$ & $160,9 \mathrm{c}$ \\
\hline & 10 & $72,2 \mathrm{a}$ & $76,4 \mathrm{a}$ & $83,1 \mathrm{a}$ & $93,1 \mathrm{a}$ & $105,9 \mathrm{a}$ & $110,4 \mathrm{a}$ & $115,0 \mathrm{a}$ & $118,1 \mathrm{a}$ \\
\hline
\end{tabular}

C.V. $(\%)=8,1$

* Letras distintas dentro de cada coluna diferem entre si pelo teste de Tukey, em 5\% de probabilidade. 
TABELA 7.Dados médios de condutividade elétrica $\left(\mu \mathrm{S} . \mathrm{cm}^{-1} \cdot \mathrm{g}^{-1}\right)$ utilizando as combinações 50 sementes/25mL, 50 sementes $/ 50 \mathrm{~mL}$ e 50 sementes $/ 75 \mathrm{~mL}$, a $30^{\circ} \mathrm{C}$, de cinco lotes de sementes das cultivares Rúcula Cultivada e Rúcula Gigante, em cada período de embebição.

\begin{tabular}{|c|c|c|c|c|c|c|c|c|c|}
\hline \multirow{2}{*}{ Cultivares } & \multirow{2}{*}{ Lotes } & \multicolumn{8}{|c|}{ Períodos de embebição (h) } \\
\hline & & 1 & 2 & 4 & 6 & 8 & 12 & 18 & 24 \\
\hline \multicolumn{10}{|c|}{50 sementes $/ 25 \mathrm{~mL} / 30^{\circ} \mathrm{C}$} \\
\hline \multirow{5}{*}{ Rúcula Cultivada } & 1 & $114,5 \mathrm{a}$ & $123,4 \mathrm{a}$ & $133,2 \mathrm{a}$ & $142,8 \mathrm{a}$ & $150,6 \mathrm{a}$ & $157,5 \mathrm{a}$ & $162,2 \mathrm{a}$ & $179,1 \mathrm{a}$ \\
\hline & 2 & $165,2 \mathrm{~b}$ & $167,2 \mathrm{~b}$ & $183,7 \mathrm{~b}$ & $173,3 \mathrm{~b}$ & $175,4 \mathrm{~b}$ & $179,4 \mathrm{~b}$ & $180,3 \mathrm{~b}$ & $189,4 \mathrm{~b}$ \\
\hline & 3 & $118,1 \mathrm{a}$ & $124,3 \mathrm{a}$ & $130,4 \mathrm{a}$ & $140,4 \mathrm{a}$ & $152,5 \mathrm{a}$ & $163,1 \mathrm{a}$ & $165,9 \mathrm{a}$ & $171,2 \mathrm{a}$ \\
\hline & 4 & $167,8 \mathrm{c}$ & $185,8 \mathrm{c}$ & $193,0 \mathrm{c}$ & $205,8 \mathrm{c}$ & $214,7 \mathrm{c}$ & $216,6 \mathrm{c}$ & $223,1 \mathrm{c}$ & $240,1 \mathrm{c}$ \\
\hline & 5 & $109,6 \mathrm{a}$ & $118,1 \mathrm{a}$ & $137,6 \mathrm{a}$ & $138,1 \mathrm{a}$ & $148,2 \mathrm{a}$ & $160,5 \mathrm{a}$ & $161,7 \mathrm{a}$ & $180,8 \mathrm{a}$ \\
\hline \multicolumn{10}{|l|}{ C.V. $(\%)=6,4$} \\
\hline \multirow{5}{*}{ Rúcula Gigante } & 6 & $104,2 \mathrm{a}$ & $108,6 \mathrm{a}$ & $124,2 \mathrm{a}$ & $140,0 \mathrm{a}$ & $149,8 \mathrm{a}$ & $162,5 \mathrm{a}$ & $172,2 \mathrm{a}$ & $176,2 \mathrm{a}$ \\
\hline & 7 & $122,1 \mathrm{~b}$ & $130,3 \mathrm{~b}$ & $136,4 \mathrm{~b}$ & $157,3 \mathrm{~b}$ & $171,4 \mathrm{~b}$ & $180,2 \mathrm{~b}$ & $192,1 \mathrm{~b}$ & $199,4 \mathrm{~b}$ \\
\hline & 8 & $126,9 \mathrm{~b}$ & $131,2 \mathrm{~b}$ & $151,0 \mathrm{c}$ & $178,4 \mathrm{c}$ & $190,2 \mathrm{c}$ & $205,6 \mathrm{c}$ & $222,3 \mathrm{c}$ & $233,9 \mathrm{c}$ \\
\hline & 9 & $125,7 \mathrm{~b}$ & $134,9 \mathrm{~b}$ & $154,5 \mathrm{c}$ & $177,9 \mathrm{c}$ & $189,0 \mathrm{c}$ & $200,2 \mathrm{c}$ & $213,0 \mathrm{c}$ & $223,5 \mathrm{c}$ \\
\hline & 10 & $103,5 \mathrm{a}$ & $111,7 \mathrm{a}$ & $121,1 \mathrm{a}$ & $137,3 \mathrm{a}$ & $151,7 \mathrm{a}$ & $161,1 \mathrm{a}$ & $178,4 \mathrm{a}$ & $169,1 \mathrm{a}$ \\
\hline \multicolumn{10}{|l|}{ C.V. $(\%)=6,8$} \\
\hline \multicolumn{10}{|c|}{50 sementes $/ 50 \mathrm{~mL} / 30^{\circ} \mathrm{C}$} \\
\hline \multirow{5}{*}{ Rúcula Cultivada } & 1 & $96,5 \mathrm{a}$ & $105,4 \mathrm{a}$ & $115,2 \mathrm{a}$ & $124,8 \mathrm{a}$ & $132,6 \mathrm{a}$ & $139,5 \mathrm{a}$ & $144,2 \mathrm{a}$ & $161,1 \mathrm{a}$ \\
\hline & 2 & $147,2 \mathrm{~b}$ & $149,2 \mathrm{~b}$ & $165,7 \mathrm{~b}$ & $155,3 \mathrm{~b}$ & $157,4 \mathrm{~b}$ & $161,4 \mathrm{~b}$ & $162,3 \mathrm{~b}$ & $171,4 \mathrm{~b}$ \\
\hline & 3 & $100,1 \mathrm{a}$ & $106,3 \mathrm{a}$ & $112,4 \mathrm{a}$ & $122,4 \mathrm{a}$ & $134,5 \mathrm{a}$ & $145,1 \mathrm{a}$ & $147,9 \mathrm{a}$ & $153,2 \mathrm{a}$ \\
\hline & 4 & $149,8 \mathrm{c}$ & $167,8 \mathrm{c}$ & $175,0 \mathrm{c}$ & $187,8 \mathrm{c}$ & $196,7 \mathrm{c}$ & $198,6 \mathrm{c}$ & $205,1 \mathrm{c}$ & $222,1 \mathrm{c}$ \\
\hline & 5 & $91,6 \mathrm{a}$ & $100,1 \mathrm{a}$ & $119,6 \mathrm{a}$ & $120,1 \mathrm{a}$ & $130,2 \mathrm{a}$ & $142,5 \mathrm{a}$ & $143,7 \mathrm{a}$ & $162,8 \mathrm{a}$ \\
\hline \multicolumn{10}{|l|}{ C.V. $(\%)=3,1$} \\
\hline \multirow{5}{*}{ Rúcula Gigante } & 6 & $88,2 \mathrm{a}$ & $92,6 \mathrm{a}$ & $108,2 \mathrm{a}$ & $124,0 \mathrm{a}$ & $133,8 \mathrm{a}$ & $146,5 \mathrm{a}$ & $156,2 \mathrm{a}$ & $160,2 \mathrm{a}$ \\
\hline & 7 & $106,1 \mathrm{~b}$ & $114,3 \mathrm{~b}$ & $120,4 \mathrm{~b}$ & $141,3 \mathrm{~b}$ & $155,4 \mathrm{~b}$ & $164,2 \mathrm{~b}$ & $176,1 \mathrm{~b}$ & $183,4 \mathrm{~b}$ \\
\hline & 8 & $110,9 \mathrm{~b}$ & $115,2 \mathrm{~b}$ & $135,0 \mathrm{c}$ & $162,4 \mathrm{c}$ & $174,2 \mathrm{c}$ & $189,6 \mathrm{c}$ & $206,3 \mathrm{c}$ & $217,9 \mathrm{c}$ \\
\hline & 9 & $109,7 \mathrm{~b}$ & $118,9 \mathrm{~b}$ & $138,5 \mathrm{c}$ & $161,9 \mathrm{c}$ & $173,0 \mathrm{c}$ & $184,2 \mathrm{c}$ & $197,0 \mathrm{c}$ & $207,5 \mathrm{c}$ \\
\hline & 10 & $87,5 \mathrm{a}$ & $95,7 \mathrm{a}$ & $105,1 \mathrm{a}$ & $121,3 \mathrm{a}$ & $135,7 \mathrm{a}$ & $145,1 \mathrm{a}$ & $162,4 \mathrm{a}$ & $153,1 \mathrm{a}$ \\
\hline \multicolumn{10}{|l|}{ C.V. $(\%)=3,3$} \\
\hline \multicolumn{10}{|c|}{50 sementes $/ 75 \mathrm{~mL} / 30^{\circ} \mathrm{C}$} \\
\hline \multirow{5}{*}{ Rúcula Cultivada } & 1 & $82,1 \mathrm{a}$ & 86,9 a & $93,8 \mathrm{a}$ & $102,3 \mathrm{a}$ & $110,2 \mathrm{a}$ & $113,6 \mathrm{a}$ & $118,9 \mathrm{a}$ & $127,2 \mathrm{a}$ \\
\hline & 2 & $118,9 \mathrm{~b}$ & $114,4 \mathrm{~b}$ & $117,4 \mathrm{~b}$ & $122,4 \mathrm{~b}$ & $123,4 \mathrm{~b}$ & $125,4 \mathrm{~b}$ & $129,5 \mathrm{~b}$ & $138,1 \mathrm{~b}$ \\
\hline & 3 & $86,4 \mathrm{a}$ & $96,8 \mathrm{ab}$ & $98,2 \mathrm{a}$ & $105,8 \mathrm{a}$ & $113,8 \mathrm{a}$ & 117,8 a & $120,4 \mathrm{a}$ & $127,6 \mathrm{a}$ \\
\hline & 4 & $119,8 \mathrm{~b}$ & $116,7 \mathrm{~b}$ & $126,1 \mathrm{c}$ & $146,9 \mathrm{c}$ & $151,7 \mathrm{c}$ & $162,9 \mathrm{c}$ & $168,3 \mathrm{c}$ & $169,8 \mathrm{c}$ \\
\hline & 5 & $84,7 \mathrm{a}$ & $85,6 \mathrm{a}$ & $96,0 \mathrm{a}$ & $103,7 \mathrm{a}$ & $110,6 \mathrm{a}$ & $112,0 \mathrm{a}$ & $117,1 \mathrm{a}$ & $125,3 \mathrm{a}$ \\
\hline \multicolumn{10}{|l|}{ C.V. $(\%)=5,2$} \\
\hline \multirow{5}{*}{ Rúcula Gigante } & 6 & $74,4 \mathrm{a}$ & $80,6 \mathrm{a}$ & 88,9 a & $100,2 \mathrm{a}$ & $109,5 \mathrm{a}$ & $116,3 \mathrm{a}$ & $122,5 \mathrm{a}$ & $129,7 \mathrm{a}$ \\
\hline & 7 & $82,1 \mathrm{~b}$ & $95,8 \mathrm{~b}$ & $105,2 \mathrm{~b}$ & $108,7 \mathrm{~b}$ & $120,4 \mathrm{~b}$ & $126,0 \mathrm{~b}$ & $133,7 \mathrm{~b}$ & $140,5 \mathrm{~b}$ \\
\hline & 8 & $91,8 \mathrm{~b}$ & $99,7 \mathrm{~b}$ & $115,4 \mathrm{c}$ & $124,8 \mathrm{c}$ & $135,7 \mathrm{c}$ & $134,9 \mathrm{c}$ & $149,6 \mathrm{c}$ & $169,8 \mathrm{c}$ \\
\hline & 9 & $90,1 \mathrm{~b}$ & $98,1 \mathrm{~b}$ & $119,6 \mathrm{c}$ & $128,3 \mathrm{c}$ & $133,6 \mathrm{c}$ & $149,7 \mathrm{c}$ & $148,3 \mathrm{c}$ & $167,4 \mathrm{c}$ \\
\hline & 10 & $75,6 \mathrm{a}$ & $80,3 \mathrm{a}$ & $88,1 \mathrm{a}$ & $101,9 \mathrm{a}$ & $112,8 \mathrm{a}$ & $116,2 \mathrm{a}$ & $123,1 \mathrm{a}$ & $127,0 \mathrm{a}$ \\
\hline
\end{tabular}

* Letras distintas dentro de cada coluna diferem entre si pelo teste de Tukey, em 5\% de probabilidade. 
Para as duas cultivares estudadas, a redução do volume de água, fixando os outros fatores avaliados (temperatura, período de embebição e número de sementes), permitiu estabelecer uma relação direta com o aumento do valor da lixiviação. Com volume de $25 \mathrm{~mL}$ foi observado os maiores valores de condutividade elétrica, e na medida em que houve aumento do volume de água utilizado, os valores da condutividade foram diminuindo gradativamente. Esses resultados concordam com os verificados por Loeffler et al. (1988), que concluiu que há maior diluição dos lixiviados em maiores volumes de água. Os resultados permitiram verificar que, de maneira geral, para todos os volumes estudados $(25,50$ e $75 \mathrm{~mL})$, houve uma relação direta com a estratificação verificada nos testes iniciais de avaliação do vigor das sementes, apontando o lote 4 como o menos vigoroso e os lotes 1 , 3 e 5 como os mais vigorosos para a Rúcula Cultivada; e os lotes 8 e 9 como os menos vigorosos para a Rúcula Gigante, destacando os lotes 6 e 10 como os de maior qualidade fisiológica. Esses resultados demonstram a possibilidade de redução no volume de água utilizado para a embebição para 50 ou $25 \mathrm{~mL}$. Porém, considerando-se que a recomendação seria utilizar 50 sementes e de acordo com os coeficientes de variação obtidos neste parâmetro, recomendase que o volume seja reduzido para $50 \mathrm{~mL}$ de água.

Com relação às temperaturas estudadas, observou-se que para as combinações empregadas utilizando-se $25^{\circ} \mathrm{C}$ e $30^{\circ} \mathrm{C}$, houve a estratificação dos lotes de maneira compatível com as avaliações iniciais de vigor das sementes. Verificouse, ainda, que o aumento dos valores da condutividade foi proporcional ao aumento da temperatura empregada, para ambas as cultivares. A elevação da temperatura de embebição provoca dano térmico às membranas, o que proporciona aumento da energia de ativação das moléculas, alternando a viscosidade da água e, por conseguinte, há aumento dos valores de condutividade elétrica (Vieira e Carvalho, 1994). As combinações utilizando temperatura de $20^{\circ} \mathrm{C}$ não permitiram a separação dos lotes de maneira correlata aos testes iniciais de avaliação do vigor das sementes, nas duas cultivares. Para a Rúcula Cultivada, verificou-se que os lotes 2 e 4 não diferiram estatisticamente entre si, tendo sido agrupados num mesmo nível de vigor, embora os testes iniciais revelaram o lote 4 como o de menor potencial fisiológico, diferindo do lote 2. Já para a Rúcula Gigante, a análise dos dados permitiu apontar o lote 8 como de menor qualidade, e os lotes 7 e 9 como intermediários, sendo que nos testes iniciais, os lotes 8 e 9 foram observados como os menos vigorosos. Esses resultados estão de acordo com Givelberg et al. (1984) que verificaram que em baixas temperaturas, o processo de reorganização das membranas se torna mais lento e o período de perda de lixiviados pelas sementes é mais longo.

De acordo com Loeffler et al. (1988) a temperatura de avaliação tem efeito significativo sobre os resultados da condutividade elétrica, onde verificaram que acréscimos ou reduções de $5^{\circ} \mathrm{C}$ na temperatura durante as leituras da condutividade elétrica, provocaram alterações significativas nos resultados. Nesse sentido, considerando que a diferença entre a temperatura de embebição e de avaliação pode influenciar os resultados, recomenda-se o uso de $25^{\circ} \mathrm{C}$ para a condução do teste de condutividade elétrica, pois essa temperatura permitiu a estratificação de ambas as cultivares quanto ao potencial fisiológico de maneira correlata aos testes iniciais de vigor, e também por ser esta a temperatura mais próxima das condições ambientais dos laboratórios de análise de sementes.

\section{CONCLUSÕES}

Diante do exposto, conclui-se que o teste de condutividade elétrica é eficiente na separação dos lotes de rúcula com relação ao potencial fisiológico, sendo que a condição mais adequada para a realização deste teste é a utilização de 50 sementes em $50 \mathrm{~mL}$ de água por 4 horas, a $25^{\circ} \mathrm{C}$.

\section{REFERÊNCIAS}

ANDRADE, R.N.; SANTOS, D.S.B.; SANTOS FILHO, B.G.; MELLO, V.D.C. Correlação entre testes de vigor em sementes de cenoura armazenadas por diferentes períodos. Pesquisa Agropecuária Gaúcha, v.1, n.2, p.153-162, 1995.

ASSOCIATION OF OFFICIAL SEED ANALYSTS. Seed vigor testing handbook. Lincoln, 1983. 93p. (Contribution, 32).

BRASIL. Ministério da Agricultura e Reforma Agrária. Regras para análise de sementes. Brasília, DF: SNDA/ DNDV/CLAV, 1992. 365p.

CARPI, V.A.F. Avaliação do potencial fisiológico de sementes de rabanete (Raphanus sativus L.). 2005. 77p. Dissertação (Mestrado) - Escola Superior de Agricultura "Luiz de Queiroz", Universidade de São Paulo, Piracicaba.

DIAS, D.C.F.S.; VIEIRA, A.N.; BHERING, M.C. Estudo dos testes de condutividade elétrica e lixiviação de potássio para avaliação do vigor de sementes de hortaliças: I. Couveflor, cebola e cenoura. In: SEMINARIO PANAMERICANO 
DE SEMILLAS, 15., Gramado, 1996. Anais... Gramado: CESM: FELAS, 1996. p.28.

DIAS, D.C.F.S.; VIEIRA, A.N.; BHERING, M.C. Condutividade elétrica e lixiviação de potássio para avaliação do vigor de sementes de hortaliças: feijão-de-vagem e quiabo. Revista Brasileira de Sementes, v.20, n.2, p.408413, 1998.

DUTRA, A.S.; VIEIRA, R.D. Teste de condutividade elétrica para a avaliação do vigor de sementes de abobrinha. Revista Brasileira de Sementes, v.28, n.2, p.117-122, 2006.

FAGIOLI, M. Relação entre a condutividade elétrica de sementes e a emergência das plântulas de milho em campo. 1997. 74f. Dissertação (Mestrado) - Faculdade de Ciências Agrárias e Veterinária, Universidade Estadual Paulista Julio de Mesquita Filho, Jaboticabal.

GIVELBERG, A.; HOROWITZ, M.; POLJAKOFFMAYBER, A. Solute leakage from Solanum nigrum L. seeds exposed to high temperatures during imbibition. Journal of Experimental Botany, v.35, n.161, p.1754-1763, 1984.

HAMPTON, J.G. Vigor testing within laboratories of the International Seed Testing Association: a survey. Seed Science and Technology, Zürich, v.20, n.1, p.199-203, 1992.

HAMPTON, J.G.; TEKRONY, B.H. Conductivity test. In: HAMPTON, J.G.; TEKRONY, B.M. (Ed.). Handbook of vigor methods. 3. ed. Zürich: ISTA, 1995. p.22-34.

INTERNATIONAL SEED TESTING ASSOCIATION. Handbook of vigour test methods. 3.ed. Zürich: ISTA, 1995. 116p.

LIMA, D. Avaliação da viabilidade e vigor de sementes de cebola (Allium cepa L.). 1993. 61p. Dissertação (Mestrado) - Faculdade de Agronomia "Eliseu Maciel", Universidade Federal de Pelotas, Pelotas.

LOEFFLER, T.M.; TEKRONY, D.M. ; EGLI, D.B. The bulk conductivity test as an indicator of soybean seed quality. Journal of Seed Technology, v.12, n.1,p.37-53,1988.

MARCOS FILHO, J. Testes de vigor: importância e utilização. In: KRZYZANOWSKI, F.C.; VIEIRA, R.D.; FRANÇA NETO, J.B. (Ed.). Vigor de sementes: conceitos e testes. Londrina: ABRATES, 1999. p.1-21.

MENDONÇA, E.A.F., RAMOS, N.P., FESSEL, S.A. Adequação da metodologia do teste de deterioração controlada para sementes de brócolis (Brassica oleracea L. - var. Itálica). Revista Brasileira de Sementes, v.25, n.1, p.18-24, 2003.

NOVEMBRE, A.D.L.C.; CARPI, V.A.F.; MARCOS
FILHO, J.; CHAMMA, H.M.C.P. Teste de condutividade elétrica para estimar o potencial fisiológico de sementes de berinjela. Horticultura Brasileira, v. 20, n.2, p.293-298, 2002.

OLIVEIRA, S.R.S.; NOVEMBRE, A.D.L.C. Teste de condutividade elétrica para as sementes de pimentão. Revista Brasileira de Sementes, v.27, n.1, p.31-36, 2005.

PANOBIANCO, M. Avaliação do potencial fisiológico de sementes de tomate. 2000. 152f. Tese (Doutorado) - Escola Superior de Agricultura "Luiz de Queiroz", Universidade de São Paulo, Piracicaba.

RODO, A.B. Avaliação do potencial fisiológico de sementes de cebola e sua relação com o desempenho das plântulas em campo. 2002. 123f. Tese (Doutorado) - Escola Superior de Agricultura "Luiz de Queiroz", Universidade de São Paulo, Piracicaba.

RODO, A.B.; TILLMANN, M.A.A.; VILLELA, F.A.; SAMPAIO, N.V. Teste de condutividade elétrica em sementes de tomate. Revista Brasileira de Sementes, v.20, n.1, p.29-38, 1998.

SÁ, M.E. de. Condutividade elétrica em sementes de tomate (Lycopersicon lycopersicum L.). Scientia Agricola, v.56, n.1, p.13-19, 1999.

TAO, J.K. Factors causing variations in the conductivity test for soybean seeds. Journal of Seed Technology, v.3,n.1, p.10-18, 1978.

TORRES, S.B. Qualidade fisiológica de sementes de pimentão (Capsicum annumm L.) através do teste de estresse hídrico. Revista Brasileira de Sementes, v.18, n.2, p.246250, 1996.

TORRES, S.B. Métodos para avaliação do potencial fisiológico de sementes de melão. 2002. 103f. Tese (Doutorado) - Escola Superior de Agricultura "Luiz de Queiroz”, Universidade de São Paulo, Piracicaba.

TORRES, S.B.; CASEIRO, R.F.; RODO, A.B.; MARCOS FILHO, J. Testes de vigor em sementes de maxixe (Cucumis anguria L.) com ênfase ao teste de condutividade elétrica. Revista Brasileira de Sementes, v.20, n.2, p.480-483, 1998.

VIEIRA, R.D.; CARVALHO, N.M. de. Testes de vigor em sementes. Jaboticabal: FUNEP, 1994. 164p.

VIEIRA, R.D.; PAIVA-AGUERO, J.A.; PERECIN, D.; BITTENCOURT, S.R.M. Correlation of electrical conductivity and other vigor tests with field emergence of soybean seedlings. Seed Science and Technology, Zürich, v.27, p.67-75, 1999. 
VIEIRA, R.D.; PENARIOL, A.L.; PERECIN, D.; PANOBIANCO, M. Condutividade elétrica e teor de água inicial das sementes de soja. Pesquisa Agropecuária Brasileira, v.37, p.1333-1338, 2002.
ZHANG, T.; HAMPTON, J.G. Does fungicide seed treatment affect bulk conductivity test results? Seed Science and Technology, v.27, n.3, p.1041-1045, 1999. 